\title{
The Navier-Stokes Equations in Space Dimension Four *
}

\author{
Vladimir Scheffer \\ Department of Mathematics, Stanford University, Stanford, California 94305, USA
}

\begin{abstract}
Solutions to the Navier-Stokes equations in four space dimensions are continuous except for a closed set whose three dimensional Hausdorff measure is finite.
\end{abstract}

\section{Section 1. Introduction and Notation}

In this paper we will prove Theorem 1.1 below. The terminology and notation in the statement of this theorem are explained in the remainder of this section.

Theorem 1.1. Let $v: R^{4} \rightarrow R^{4}$ be a measurable function such that $\int|v(x)|^{2} d x<\infty$ and $\operatorname{div}(v)=0$. Then there exists a measurable function $u: R^{4} \times(0, \infty) \rightarrow R^{4}$ such that

$$
\int_{0}^{\infty} \int|u(x, t)|^{3} d x d t<\infty
$$

$u$ is a weak solution to the Navier-Stokes equations of incompressible fluid flow with initial condition $v$, and the following property holds: There exists a set $A \subset R^{4} \times(0, \infty)$ such that
a) $A \cap\left(R^{4} \times[\varepsilon, \infty)\right)$ is compact for every $\varepsilon>0$,
b) the 3-dimensional Hausdorff measure of $A$ is finite, and
c) the restriction of $u$ to the complement of $A$ is a continuous function.

Notation. Hausdorff measure is defined in Section 2 (Definition 2.8). If $X$ and $Y$ are euclidean spaces then $C^{\infty}(X, Y)$ is the set of all infinitely differentiable functions from $X$ into $Y$, and $C_{0}^{\infty}(X, Y)$ is the set of all functions in $C^{\infty}(X, Y)$ with compact support. If $f$ is a $C^{\infty}$ function defined on an open subset of $R^{4} \times R\left(R^{4}\right.$ should be thought of as space and $R$ as time) then $D_{i} f, D_{i j} f$, and $D_{i j k} f$ are the partial derivatives $\left(\partial / \partial x_{i}\right) f,\left(\partial^{2} / \partial x_{i} \partial x_{j}\right) f$, and $\left(\partial^{3} / \partial x_{i} \partial x_{j} \partial x_{k}\right) f$ with respect to the variables $x_{1}, x_{2}, x_{3}, x_{4}$ of $R^{4}$. The partial derivative of $f$ with respect to the $R$ variable of

* This research was supported in part by the National Science Foundation Grant MCS 75-23332A02 
$R^{4} \times R$ is denoted by $D_{t} f$ (the time derivative). We also set $\Delta f=\sum_{i=1}^{4} D_{i i} f=D_{i i} f$ (repeated indices are always summed), $\operatorname{div}(f)=\sum_{i=1}^{4} D_{i} f_{i}=D_{i} f_{i}$ if the range of $f$ is $R^{4}$, and $\nabla(f)=\left(D_{1} f, D_{2} f, D_{3} f, D_{4} f\right)$. Note that the time derivative is not included in the definitions of $\Delta$, div, and $\nabla$. Analogous definitions are made for functions with domain $R^{4}$. If $v: R^{4} \rightarrow R^{4}$ is square integrable then $\operatorname{div}(v)=0$ is interpreted in the distribution sense. We also set $[a, b)=\{t: a \leqq t<b\}$. Closed and open intervals are denoted $[a, b]$ and $(a, b)$, respectively.

Suppose $v: R^{4} \rightarrow R^{4}$ is square integrable with $\operatorname{div}(v)=0$. A measurable function $u: R^{4} \times(0, \infty) \rightarrow R^{4}$ satisfying $\left.\int_{0}^{\infty} \int u(x, t)\right|^{3} d x d t<\infty$ is called a weak solution to the Navier-Stokes equations of incompressible fluid flow with initial condition $v$ when (1.1) and (1.2) are satisfied:

$$
\begin{aligned}
& \int_{0}^{\infty} \int_{i} u_{i}(x, t) D_{i} \phi(x, t) d x d t=0 \quad \text { if } \quad \phi \in C_{0}^{\infty}\left(R^{4} \times R, R\right) \\
& \int v_{i}(x) \phi_{i}(x, 0) d x+\int_{0}^{\infty} \int u_{i}(x, t)\left(D_{t} \phi_{i}+\Delta \phi_{i}\right)(x, t) d x d t \\
& \quad+\int_{0}^{\infty} \int u_{j}(x, t) u_{i}(x, t) D_{j} \phi_{i}(x, t) d x d t=0 \quad \text { if } \quad \phi \in C_{0}^{\infty}\left(R^{4} \times R, R^{4}\right), \operatorname{div}(\phi)=0 .
\end{aligned}
$$

Observe that Hölder's inequality, the integrability of $|u|^{3}$, and the integrability of $|v|^{2}$ imply that the integrals in (1.1) and (1.2) make sense.

If $a \in R^{4}$ and $0<r<\infty$ we set $B(a, r)=\left\{x \in R^{4}:|x-a| \leqq r\right\}$. If $X$ is a measure space and $Y$ is a euclidean space then $L^{p}(X, Y)$ is the Lebesgue $L^{p}$ space of functions with domain $X$ and range $Y$. The $L^{p}$ norm is denoted \|\|$_{p}$. The norm | is always euclidean norm. If $f$ is a $C^{\infty}$ function defined on an open subset of $R^{4} \times R$ or $R^{4}$ then $\nabla f$ (see above) will also be denoted $D f$. In addition, $D^{2} f$ and $D^{3} f$ will be the vector valued functions with components $D_{i j} f$ and $D_{i j k} f$, respectively $(i, j, k \in\{1,2,3,4\})$. If $\phi \in C_{0}^{\infty}(X, Y)$ (see above) then $\operatorname{spt}(\phi)$ is the closure of $\{x: \phi(x) \neq 0\}$. If $f$ is a function defined on a subset of $R^{4} \times R$ and $g, k$ are functions defined on $R^{4}$ then we set

$$
\begin{aligned}
(f * k)(x, t) & =\int f(y, t) k(x-y) d y, \\
(g * k)(x) & =\int g(y) k(x-y) d y
\end{aligned}
$$

whenever the integrals make sense. If $0<t<\infty$ we define $H_{t}: R^{4} \rightarrow R$ by

$$
H_{t}(x)=(4 \pi t)^{-2} \exp \left(-|x|^{2} / 4 t\right) \text {. }
$$

If $t$ has a complicated form we will write

$$
H[t]=H_{t} .
$$

We also define $K: R^{4}-\{0\} \rightarrow R$ by

$$
K(x)=-\left(4 \pi^{2}|x|^{2}\right)^{-1} .
$$


An absolute constant is a finite positive constant that does not depend on any of the parameters in this paper. The symbol $C$ will always denote an absolute constant, and the value of $C$ may change from one line to the next (e.g. $2 C \leqq C$ ). The symbols $C_{1}, C_{2}, C_{3}, \ldots$ will also denote absolute constants, but their values will not change in the course of the paper.

We fix $v$ satisfying the hypothesis of Theorem 1.1 and set

$$
L=\int|v(x)|^{2} d x \text {. }
$$

See [4], [5] and [6] for theorems on the Navier-Stokes equations in 3-dimensional space. This program was inspired by the work of Mandelbrot [3] and Almgren [1].

\section{Section 2. Preliminary Results Involving Hausdorff Measure}

Throughout this section we fix a positive real number $D$ and functions $f$ and $f_{n}$ in $L^{3}\left(R^{4} \times[0, \infty), R^{4}\right)$ for $n \in\{1,2,3, \ldots\}$ such that $\left\|f_{n}\right\|_{3}^{3} \leqq D$ and the sequence $f_{n}$ converges to $f$ weakly in $L^{3}$.

Definition 2.1. If $a \in R^{4}, 0<b<\infty, m$ is an integer satisfying $2^{-2 m} \leqq b$, and $n \in\{1,2,3, \ldots\}$ then we set

$$
\begin{aligned}
& A(a, b, m, n)=2^{m}\left(\int_{b-2^{-2 m}}^{b} \int_{R^{4}}\left|f_{n}(x, t)\right|^{3}\left(|x-a|+2^{-m}\right)^{-5} d x d t\right), \\
& B(a, b, m, n)=2^{6 m}\left(\int_{b-2^{-2 m}}^{b} \int_{B\left(a, 2^{-m}\right)}\left|f_{n}(x, t)\right|^{3} d x d t\right) .
\end{aligned}
$$

Definition 2.2. Whenever $0<M<\infty$ the following statement will be known as property $P(M)$ : If $n_{1}, n_{2}, n_{3}, \ldots$ is an increasing sequence of positive integers, $p$ and $q$ are integers, $p<q, a \in R^{4}, 0<b<\infty$, and $2^{-2 p} \leqq b$ then

$$
\begin{aligned}
& \liminf _{k \rightarrow \infty} B\left(a, b, q, n_{k}\right) \\
& \quad \leqq M\left(\liminf _{k \rightarrow \infty}\left(A\left(a, b, p, n_{k}\right)+\left(2^{-p} A\left(a, b, p, n_{k}\right)+\sum_{m=p}^{q-1} 2^{-m} B\left(a, b, m, n_{k}\right)\right)^{3 / 2}\right)\right) .
\end{aligned}
$$

From (2.1) and (2.2) we conclude that there exists an absolute constant $C_{1}$ such that

$$
B(a, b, m, n) \leqq C_{1}(A(a, b, m, n)) .
$$

We use $C_{1}$ in the following lemma :

Lemma 2.3. For every positive real number $M$ there exists $\varepsilon>0$ such that the following holds: If property $P(M)$ is satisfied, $a \in R^{4}, 0<b<\infty, p$ is an integer, $2^{-2 p}$ $\leqq b$, and

$$
\liminf _{n \rightarrow \infty} A(a, b, p, n) \leqq \varepsilon 2^{3 p}
$$


then

$$
\liminf _{n \rightarrow \infty} B(a, b, q, n) \leqq \varepsilon\left(M+C_{1}+1\right) 2^{3 p}
$$

for all integers $q$ satisfying $q \geqq p$.

Proof. We choose $\varepsilon>0$ such that

$$
M\left(1+2\left(M+C_{1}+1\right)\right)^{3 / 2} \varepsilon^{3 / 2} \leqq \varepsilon .
$$

There is an increasing sequence $n_{1}, n_{2}, n_{3}, \ldots$ of positive integers such that

$$
\liminf _{n \rightarrow \infty} A(a, b, p, n)=\lim _{k \rightarrow \infty} A\left(a, b, p, n_{k}\right) .
$$

Using the Cantor diagonal process and passing to a subsequence, we may assume

$$
\lim _{k \rightarrow \infty} B\left(a, b, m, n_{k}\right) \quad \text { exists if } \quad m \geqq p .
$$

It suffices to prove

$$
\lim _{k \rightarrow \infty} B\left(a, b, m, n_{k}\right) \leqq \varepsilon\left(M+C_{1}+1\right) 2^{3 p}
$$

if $m \geqq p$. We proceed by induction on $m$. If $m=p$ then (2.9) follows from (2.7), (2.8), (2.3), and (2.4). Now suppose that (2.9) holds for $m=p, p+1, \ldots, q-1$. Then from (2.8), property $P(M)$, Definition $2.2,(2.7),(2.8),(2.4)$, and (2.6) we obtain

$$
\begin{aligned}
\lim _{k \rightarrow \infty} B\left(a, b, q, n_{k}\right) & \leqq \\
\leqq & M\left(\liminf _{k \rightarrow \infty}\left(A\left(a, b, p, n_{k}\right)+\left(2^{-p} A\left(a, b, p, n_{k}\right)+\sum_{m=p}^{q-1} 2^{-m} B\left(a, b, m . n_{k}\right)\right)^{3 / 2}\right)\right) \\
= & M\left(\lim _{k \rightarrow \infty} A\left(a, b, p, n_{k}\right)\right) \\
& +M\left(2^{-p}\left(\lim _{k \rightarrow \infty} A\left(a, b, p, n_{k}\right)\right)+\sum_{m=p}^{q-1} 2^{-m}\left(\lim _{k \rightarrow \infty} B\left(a, b, m, n_{k}\right)\right)\right)^{3 / 2} \\
\leqq & M \varepsilon 2^{3 p}+M\left(2^{-p} \varepsilon 2^{3 p}+\sum_{m=p}^{q-1} 2^{-m} \varepsilon\left(M+C_{1}+1\right) 2^{3 p}\right)^{3 / 2} \\
& <M \varepsilon 2^{3 p}+M\left(\varepsilon 2^{2 p}+2^{-p+1} \varepsilon\left(M+C_{1}+1\right) 2^{3 p}\right)^{3 / 2} \\
= & M \varepsilon 2^{3 p}+M \varepsilon^{3 / 2} 2^{3 p}\left(1+2\left(M+C_{1}+1\right)\right)^{3 / 2} \leqq M \varepsilon 2^{3 p}+\varepsilon 2^{3 p}<\varepsilon\left(M+C_{1}+1\right) 2^{3 p} .
\end{aligned}
$$

The lemma has been proved.

Lemma 2.4. There exists an absolute constant $C_{2}$ with the following property: If $a \in R^{4}, 0<b<\infty, p$ is an integer, and $2^{-2 p+2} \leqq b$ then

$$
A(c, d, p, n) \leqq C_{2}(A(a, b, p-1, n))
$$

whenever $c \in R^{4},\left|a_{i}-c_{i}\right| \leqq 2^{-p-1}$ for $i \in\{1,2,3,4\}$, and $b-2^{-2 p} \leqq d \leqq b$.

Proof. This follows easily from (2.1).

Definition 2.5. Let $p$ be an integer. We let $Z(p)$ be the collection of all points $(a, b) \in R^{4} \times\left[2^{-2 p+2}, \infty\right)$ which satisfy the following: $a=\left(i_{1} 2^{-p}, i_{2} 2^{-p}, i_{3} 2^{-p}, i_{4} 2^{-p}\right)$ where $i_{1}, i_{2}, i_{3}, i_{4}$ are integers, and $b=j 2^{-2 p}$ where $j$ is an integer satisfying $j \geqq 4$. 
In the following lemma we use the function $f$ given at the start of this section.

Lemma 2.6. Suppose $M$ is a positive real number, property $P(M)$ holds, $\varepsilon$ is as in Lemma 2.3, $p$ is an integer, $p \geqq 0,(a, b) \in Z(p)$,

$$
\liminf _{n \rightarrow \infty} A(a, b, p-1, n) \leqq C_{2}^{-1} \varepsilon 2^{3 p}
$$

(see Lemma 2.4), and

$$
Q=\left\{(c, d) \in R^{4} \times R:\left|a_{i}-c_{i}\right| \leqq 2^{-p-1} \quad \text { for } \quad i \in\{1,2,3,4\},\right.
$$

and $\left.b-2^{-2 p} \leqq d \leqq b\right\}$.

Then $|f(c, d)|^{3} \leqq C \varepsilon\left(M+C_{1}+1\right) 2^{3 p}$ for almost every $(c, d) \in Q$.

Proof. From Definition 2.5, Lemma 2.4, and the hypotheses we obtain

$$
\liminf _{n \rightarrow \infty} A(c, d, p, n) \leqq C_{2}\left(\liminf _{n \rightarrow \infty} A(a, b, p-1, n)\right) \leqq \varepsilon 2^{3 p}
$$

if $(c, d) \in Q$. Hence Lemma 2.3 yields

$$
\liminf _{n \rightarrow \infty} B(c, d, q, n) \leqq \varepsilon\left(M+C_{1}+1\right) 2^{3 p}
$$

if $q \geqq p$ and $(c, d) \in Q$. Hence (2.2) and the assumption that $f_{n}$ converges to $f$ weakly in $L^{3}$ imply

$$
2^{6 q}\left(\int_{d-2^{-2 q}}^{d} \int_{B\left(c, 2^{-q}\right)}|f(x, t)|^{3} d x d t\right) \leqq \varepsilon\left(M+C_{1}+1\right) 2^{3 p}
$$

if $q$ is an integer, $q \geqq p$, and $(c, d) \in Q$. We set

$$
Q^{\prime}=\left\{(y, s) \in R^{4} \times R:\left|a_{i}-y_{i}\right|<2^{-p-1} \text { for } i \in\{1,2,3,4\},\right.
$$

and $\left.b-2^{-2 p}<s<b\right\}$.

Let $(y, s) \in Q^{\prime}$. Choose an integer $q^{\prime}$ such that $q^{\prime} \geqq p,\left|a_{i}-y_{i}\right|+2^{-q^{\prime}} \leqq 2^{-p-1}$ for $i \in\{1,2,3,4\}, b-2^{-2 p} \leqq s-2^{-q^{\prime}}$, and $s+2^{-q^{\prime}} \leqq b$. Let $q$ be an integer such that $q \geqq q^{\prime}$. We set

$$
Q_{j}=\left\{(x, t) \in R^{4} \times R:|x-y| \leqq 2^{-q},\left(s+j 2^{-2 q}\right)-2^{-2 q} \leqq t \leqq s+j 2^{-2 q}\right\}
$$

whenever $j$ is an integer and $1-2^{q} \leqq j \leqq 2^{q}$. We also set

$$
Q^{\prime \prime}=\left\{(x, t) \in R^{4} \times R:|x-y| \leqq 2^{-q},|s-t| \leqq 2^{-q}\right\} .
$$

From $q \geqq q^{\prime} \geqq p \geqq 0$ we conclude that $1-2^{q}$ and $2^{q}$ are integers and satisfy $1-2^{q}$ $<2^{q}$. Hence (2.13) and (2.14) yield

$$
\int_{Q^{\prime \prime}}|f|^{3}=\sum_{j}\left(\int_{Q_{j}}|f|^{3}\right) \text { where the sum is taken over } 1-2^{q} \leqq j \leqq 2^{q} .
$$

Using (2.15), (2.13), (2.11) with $(c, d)=\left(y, s+j 2^{-2 q}\right)$, the properties of $q^{\prime}$, and (2.10) we obtain

$$
2^{6 q}\left(\int_{Q^{\prime \prime}}|f|^{3}\right) \leqq 2^{q+1}(\varepsilon)\left(M+C_{1}+1\right) 2^{3 p}
$$


For every $r>0$ we set

$$
B(y, s, r)=\left\{(x, t) \in R^{4} \times R:|y-x|^{2}+|s-t|^{2} \leqq r^{2}\right\}
$$

and we let $m$ be the Lebesgue measure on $R^{4} \times R$. From (2.17) and (2.14) we conclude $B\left(y, s, 2^{-q}\right) \subset Q^{\prime \prime}$. Hence (2.16) and (2.17) yield

$$
\left(m\left(B\left(y, s, 2^{-q}\right)\right)\right)^{-1}\left(\int_{B\left(y, s, 2^{-q}\right)}|f|^{3}\right) \leqq C \varepsilon\left(M+C_{1}+1\right) 2^{3 p} .
$$

Since $|f|^{3}$ is an integrable function, (2.17) yields

$$
\lim _{q \rightarrow \infty}\left(m\left(B\left(y, s, 2^{-q}\right)\right)\right)^{-1}\left(\int_{B\left(y, s, 2^{-q}\right)}|f|^{3}\right)=|f(y, s)|^{3}
$$

for almost every $(y, s) \in Q^{\prime}$. Now (2.18), (2.19), and the fact that $Q^{\prime}$ is almost all of $Q$ yield the conclusion of the lemma.

In the lemma below we use Definition 2.5 and the number $D$ that was fixed at the start of this section.

Lemma 2.7. There exists an absolute constant $C_{3}$ which satisfies the following: If $p$ is an integer then

$$
\sum_{(a, b) \in Z(p)} \liminf _{n \rightarrow \infty} A(a, b, p-1, n) \leqq C_{3} 2^{6 p} D .
$$

Proof. For each $(a, b) \in Z(p)$ (see Definition 2.5) we define $\phi_{a, b}: R^{4} \times[0, \infty) \rightarrow R$ by

$$
\phi_{a, b}(x, t)=\left(|x-a|+2^{-p+1}\right)^{-5} \quad \text { if } \quad b-2^{-2 p+2} \leqq t \leqq b,
$$

$\phi_{a, b}(x, t)=0 \quad$ otherwise.

We have

$$
\left\|\sum_{(a, b) \in Z(p)} \phi_{a, b}\right\|_{\infty} \leqq C 2^{5 p} .
$$

From (2.1) we obtain

$$
\begin{aligned}
& A(a, b, p-1, n)=2^{p-1}\left(\int\left|f_{n}\right|^{3} \phi_{a, b}\right) . \text { Hence } \\
& \sum_{(a, b) \in Z(p)} A(a, b, p-1, n) \leqq C 2^{6 p}\left\|f_{n}\right\|_{3}^{3} \leqq C 2^{6 p} D .
\end{aligned}
$$

The conclusion follows from the inequality $\liminf _{n \rightarrow \infty}\left(a_{n}\right)+\liminf _{n \rightarrow \infty}\left(b_{n}\right) \leqq \liminf _{n \rightarrow \infty}\left(a_{n}\right.$ $\left.+b_{n}\right)$.

Definition 2.8. For any nonempty subset $B$ of $R^{4} \times R$ we define

$$
\operatorname{diam}(B)=\sup \left\{\left(|a-c|^{2}+|b-d|^{2}\right)^{1 / 2}:(a, b) \in B \quad \text { and } \quad(c, d) \in B\right\} .
$$

Let $A$ be a subset of $R^{4} \times R$. For every $\delta>0$ we define $\phi_{\delta}(A)$ to be the infimum of all numbers of the form

$$
\sum_{i=1}^{\infty}(4 / 3) \pi\left(2^{-1} \operatorname{diam}\left(A_{i}\right)\right)^{3}
$$

where $A_{i}$ is a nonempty subset of $R^{4} \times R, A \subset \bigcup_{i=1}^{\infty} A_{i}$, and diam $\left(A_{i}\right) \leqq \delta$. Observe that 
$\phi_{\delta}(A) \geqq \phi_{\eta}(A)$ if $\delta \leqq \eta$. This allows us to define $H^{3}(A)=\lim _{\delta \rightarrow 0} \phi_{\delta}(A)$. The number $H^{3}(A)$ is called the 3-dimensional Hausdorff measure of $A$. There is an extensive treatment of Hausdorff measure in [2].

Lemma 2.9. Suppose $p$ is an integer, $p \geqq 0, M$ is a positive real number, property $P(M)$ holds, and $\varepsilon$ is as in Lemma 2.3. Then there exists a set $A_{p}$ such that

1) $A_{p} \subset R^{4} \times\left[3\left(2^{-2 p}\right), \infty\right)$ and $A_{p}$ is compact,

2) $\phi_{\delta}\left(A_{p}\right) \leqq C \varepsilon^{-1} D$ if $\delta \geqq 5^{1 / 2} 2^{-p}$,

3) $|f(x, t)|^{3} \leqq C \varepsilon\left(M+C_{1}+1\right) 2^{3 p}$ for almost every $(x, t)$ that satisfies the conditions $(x, t) \in R^{4} \times\left[3\left(2^{-2 p}\right), \infty\right)$ and $(x, t) \notin A_{p}$.

Proof. For each point $(a, b) \in Z(p)$ (see Definition 2.5) we set

$$
Q(a, b)=\left\{(c, d) \in R^{4} \times R:\left|a_{i}-c_{i}\right| \leqq 2^{-p-1} \text { for } i \in\{1,2,3,4\},\right.
$$

and $\left.b-2^{-2 p} \leqq d \leqq b\right\}$.

From Definition 2.5 we obtain

$$
\cup\{Q(a, b):(a, b) \in Z(p)\}=R^{4} \times\left[3\left(2^{-2 p}\right), \infty\right) .
$$

We set

$$
\begin{aligned}
& Y(p)=\left\{(a, b) \in Z(p): \liminf _{n \rightarrow \infty} A(a, b, p-1, n) \geqq C_{2}^{-1} \varepsilon 2^{3 p}\right\}, \\
& A_{p}=\cup\{Q(a, b):(a, b) \in Y(p)\} .
\end{aligned}
$$

From (2.22) and Lemma 2.7 we obtain

$$
\begin{aligned}
& \text { (cardinality }(Y(p))) C_{2}^{-1} \varepsilon 2^{3 p}=\sum_{(a, b) \in Y(p)} C_{2}^{-1} \varepsilon 2^{3 p} \\
& \leqq \sum_{(a, b) \in Y(p)} \liminf _{n \rightarrow \infty} A(a, b, p-1, n) \\
& \leqq \sum_{(a, b) \in Z(p)} \liminf _{n \rightarrow \infty} A(a, b, p-1, n) \leqq C_{3} 2^{6 p} D<\infty .
\end{aligned}
$$

We conclude from (2.24) that $Y(p)$ is a finite set. Combining this with (2.20) and (2.23) we obtain that $A_{p}$ is compact. This fact and (2.21) yield part 1). From (2.20) and $p \geqq 0$ we conclude (see Definition 2.8)

$$
\operatorname{diam}(Q(a, b))=\left(4\left(2^{-2 p}\right)+2^{-4 p}\right)^{1 / 2} \leqq 5^{1 / 2} 2^{-p} .
$$

Combining (2.24) and (2.25) we obtain

$$
\sum_{(a, b) \in Y(p)}(4 / 3) \pi\left(2^{-1} \operatorname{diam}(Q(a, b))\right)^{3} \leqq C \varepsilon^{-1} D .
$$

Now the countability of $Z(p),(2.23),(2.25)$, and (2.26) yield part 2) of the lemma. From (2.21) and (2.23) we obtain

$$
\left(R^{4} \times\left[3\left(2^{-2 p}\right), \infty\right)\right)-A_{p} \subset \cup\{Q(a, b):(a, b) \in Z(p)-Y(p)\} .
$$

Take $(a, b) \in Z(p)$ such that $(a, b) \notin Y(p)$. Then (2.22) yields

$$
\liminf _{n \rightarrow \infty} A(a, b, p-1, n)<C_{2}^{-1} \varepsilon 2^{3 p} .
$$


Hence Lemma 2.6 and (2.20) yield

$$
|f(x, t)|^{3} \leqq C \varepsilon\left(M+C_{1}+1\right) 2^{3 p} \quad \text { for almost every } \quad(x, t) \in Q(a, b)
$$

if $(a, b) \in Z(p)-Y(p)$. Finally, (2.27), (2.28), and the countability of $Z(p)$ yield part 3) of the lemma.

\section{Section 3. Estimates on Vector Fields}

Throughout this section we fix a positive real number $\zeta$ and a $C^{\infty}$ function $w: R^{4} \rightarrow R^{4}$ such that $\operatorname{div}(w)=0, \int|w|^{2}<\infty$, and $\int|D w|^{2}<\infty$.

Lemma 3.1. $\int|w|^{3} \leqq C\left(\int|D w|^{2}\right)\left(\int|w|^{2}\right)^{1 / 2}$.

Proof. The Schwarz inequality and the case $n=4, p=2, q=4$ of [7, Line 9, p. 127] yield

$$
\int|w|^{3}=\int|w|^{2}|w| \leqq\left(\int|w|^{4}\right)^{1 / 2}\left(\int|w|^{2}\right)^{1 / 2} \leqq C\left(\int|D w|^{2}\right)\left(\int|w|^{2}\right)^{1 / 2} .
$$

Lemma 3.2. If $a \in R^{4}, t>0$, and $t^{1 / 2} \leqq r<\infty$ then (see (1.3))

$$
\int\left|\left(w * H_{t}\right)(x)\right|^{3}(|x-a|+r)^{-5} d x \leqq C\left(\int|w(x)|^{3}(|x-a|+r)^{-5} d x\right) .
$$

Proof. For every $i \in\{0,1,2, \ldots\}$ we define $h_{i}: R^{4} \rightarrow R$ as follows : If $i>0$ then $h_{i}(x)=1$ for every $x \in B\left(0,2^{i} r\right)-B\left(0,2^{i-1} r\right)$ and $h_{i}(x)=0$ otherwise. We set $h_{0}(x)=1$ if $x \in B(0, r)$ and $h_{0}(x)=0$ otherwise. For every $i$ we use Young's inequality to obtain

$$
\begin{aligned}
& \int\left|\left(w * H_{t} h_{i}\right)(x)\right|^{3}(|x-a|+r)^{-5} d x \\
& \leqq C\left(\sum_{j=0}^{\infty} \int_{B\left(a, 2^{j} r\right)}\left|w * H_{t} h_{i}\right|^{3}\left(2^{j} r\right)^{-5}\right) \\
& \leqq C\left(\sum_{j=0}^{\infty}\left(\int_{B\left(a, 2^{j} r+2^{i} r\right)}|w|^{3}\left(2^{j} r\right)^{-5}\right)\left\|H_{t} h_{i}\right\|_{1}^{3}\right) \\
& \leqq C\left(\sum_{j=0}^{i}\left(\int_{B\left(a, 2^{i+1} r\right)}|w|^{3}\left(2^{j} r\right)^{-5}\right)\left\|H_{t} h_{i}\right\|_{1}^{3}\right) \\
& +C\left(\sum_{j=i+1}^{\infty}\left(\int_{B\left(a, 2^{j+1} r\right)}|w|^{3}\left(2^{j} r\right)^{-5}\right)\left\|H_{t} h_{i}\right\|_{1}^{3}\right) \\
& \leqq C\left(\int_{B\left(a, 2^{i+1} r\right)}|w|^{3}\left(2^{i} r\right)^{-5} 2^{5 i}\right)\left\|H_{t} h_{i}\right\|_{1}^{3} \\
& +C\left(\sum_{j=i+1}^{\infty}\left(\int_{B\left(a, 2^{\left.j+1_{r}\right)}\right.}|w|^{3}\left(2^{j} r\right)^{-5} 2^{5 i}\right)\left\|H_{t} h_{i}\right\|_{1}^{3}\right) \\
& \leqq C\left(\sum_{j=0}^{\infty}\left(\int_{B\left(a, 2^{j+1} r\right)}|w|^{3}\left(2^{j} r\right)^{-5}\right) 2^{5 i}\left\|H_{t} h_{i}\right\|_{1}^{3}\right) \\
& \leqq C\left(\int|w(x)|^{3}(|x-a|+r)^{-5} d x\right) 2^{5 i}\left\|H_{t} h_{i}\right\|_{1}^{3} .
\end{aligned}
$$


We define a measure $m$ on $R^{4}$ by $m(E)=\int_{E}(|x-a|+r)^{-5} d x$. Minkowski's inequality and (3.1) yield

$$
\begin{aligned}
& \left(\int\left|\left(w * H_{t}\right)(x)\right|^{3}(|x-a|+r)^{-5} d x\right)^{1 / 3}=\left(\int\left|w * H_{t}\right|^{3} d m\right)^{1 / 3} \\
& \quad \leqq \sum_{i=0}^{\infty}\left(\int\left|w * H_{t} h_{i}\right|^{3} d m\right)^{1 / 3} \\
& \quad=\sum_{i=0}^{\infty}\left(\int\left|\left(w * H_{t} h_{i}\right)(x)\right|^{3}(|x-a|+r)^{-5} d x\right)^{1 / 3} \\
& \quad \leqq C\left(\int|w(x)|^{3}(|x-a|+r)^{-5} d x\right)^{1 / 3}\left(\sum_{i=0}^{\infty} 2^{5 i / 3}\left\|H_{t} h_{i}\right\|_{1}\right) .
\end{aligned}
$$

From $t^{1 / 2} \leqq r$ we obtain $\sum_{i=0}^{\infty} 2^{5 i / 3}\left\|H_{t} h_{i}\right\|_{1} \leqq C$. This inequality and (3.2) complete the proof of the lemma.

Definition 3.3. We define $f: R^{4} \rightarrow R^{4}$ by $f_{i}(x)=\left(w_{j} * H_{\zeta}\right)(x) D_{j} w_{i}(x)$. We obtain

$$
\|f\|_{2} \leqq\left\|w * H_{\zeta}\right\|_{\infty}\|D w\|_{2} \leqq\|w\|_{2}\left\|H_{\zeta}\right\|_{2}\|D w\|_{2}<\infty .
$$

It is elementary that every $\phi \in C_{0}^{\infty}\left(R^{4}, R^{4}\right)$ has an orthogonal decomposition (consisting of a divergence free vector field and a gradient vector field) $\phi=\phi^{\prime}+\phi^{\prime \prime}$ in the Hilbert space $L^{2}\left(R^{4}, R^{4}\right)$ where $[$ see $(1.5)] \phi^{\prime \prime}=(\nabla \operatorname{div}(\phi)) * K=\operatorname{div}(\phi) * \nabla K[$ so that $\left.\Delta \phi^{\prime \prime}=\nabla \operatorname{div}(\phi)\right]$. We conclude

$$
\|\phi-((\nabla \operatorname{div}(\phi)) * K)\|_{2}=\left\|\phi^{\prime}\right\|_{2} \leqq\|\phi\|_{2} .
$$

Hence we can use (3.3) to construct $g \in L^{2}\left(R^{4}, R^{4}\right)$ such that (3.4) and (3.5) hold:

$$
\begin{aligned}
& \|g\|_{2} \leqq\|f\|_{2}, \\
& \int g \cdot \phi=\int f \cdot(\phi-((\nabla \operatorname{div}(\phi)) * K)) \text { if } \phi \in C_{0}^{\infty}\left(R^{4}, R^{4}\right) .
\end{aligned}
$$

Lemma 3.4. If $a \in R^{4}, \zeta^{1 / 2} \leqq r<\infty, \phi \in C_{0}^{\infty}\left(R^{4}, R\right)$, and $\operatorname{spt}(\phi) \subset B(a, r)$ then

$$
\begin{aligned}
& \left|\int g_{i}(x) w_{i}(x) \phi(x) d x\right| \\
& \leqq C\left(\|D \phi\|_{\infty}+r^{-1}\|\phi\|_{\infty}\right) r^{5}\left(\int|w(x)|^{3}(|x-a|+r)^{-5} d x\right) .
\end{aligned}
$$

Proof. We define $J: R^{4} \rightarrow R$ by

$$
J(x)=(|x-a|+r)^{-5} .
$$

Let $\varepsilon>0$ such that $4 \varepsilon<r$ and $4 \varepsilon<r^{-1}$. We construct $C^{\infty}$ functions $\alpha^{\prime}, \beta^{\prime}$, $\gamma^{\prime}$ with domain $R^{4}$ and range $[0,1]$ such that the following conditions are satisfied: $\alpha^{\prime}(x)=1$ if $x \in B(0, \varepsilon), \alpha^{\prime}(x)=0$ if $x \notin B(0,2 \varepsilon), \varepsilon\left\|D \alpha^{\prime}\right\|_{\infty}+\varepsilon^{2}\left\|D^{2} \alpha^{\prime}\right\|_{\infty} \leqq C, \beta^{\prime}(x)=1$ if $x \in B(0, r), \beta^{\prime}(x)=0$ if $x \notin B(0,2 r), r\left\|D \beta^{\prime}\right\|_{\infty}+r^{2}\left\|D^{2} \beta^{\prime}\right\|_{\infty}+r^{3}\left\|D^{3} \beta^{\prime}\right\|_{\infty} \leqq C, \gamma^{\prime}(x)=1$ if $x \in B\left(0, \varepsilon^{-1}\right), \gamma^{\prime}(x)=0$ if $x \notin B\left(0,2 \varepsilon^{-1}\right), \varepsilon^{-1}\left\|D \gamma^{\prime}\right\|_{\infty}+\varepsilon^{-2}\left\|D^{2} \gamma^{\prime}\right\|_{\infty}+\varepsilon^{-3}\left\|D^{3} \gamma^{\prime}\right\|_{\infty} \leqq C$. We define functions $\alpha, \beta, \gamma, \delta$ with domain $R^{4}$ and range $[0,1]$ as follows: $\alpha=\alpha^{\prime}$, $\beta=\beta^{\prime}-\alpha^{\prime}, \gamma=\gamma^{\prime}-\beta^{\prime}, \delta=1-\gamma^{\prime}$. We have

$$
\begin{aligned}
& \alpha+\beta+\gamma+\delta=1, \\
& \|\alpha K\|_{1} \leqq C \varepsilon^{2},\left|D^{3}(\gamma K)(x)\right| \leqq C(|x|+r)^{-5},\|D(\delta K)\|_{2} \leqq C \varepsilon .
\end{aligned}
$$


Since $\Delta K(x)=0$ for every $x \neq 0$, we have $\Delta(\beta K)(x)=0$ for every $x \in B(0, r)-B(0,2 \varepsilon)$. We conclude

$$
\|\Delta(\beta K)\|_{1} \leqq C \text {. }
$$

We use Definition 3.3, $\operatorname{div}(w)=0$, the Schwarz inequality, Young's inequality, and (3.8) to obtain

$$
\begin{aligned}
& \left|\int f_{i} D_{i}(\operatorname{div}(w \phi) * \alpha K)\right| \\
= & \left|\int f_{i} D_{i}\left(\left(w_{j} D_{j} \phi\right) * \alpha K\right)\right| \\
= & \left|\int f_{i}\left(D_{i}\left(w_{j} D_{j} \phi\right) * \alpha K\right)\right| \\
\leqq & \|f\|_{2}\left\|D\left(w_{j} D_{j} \phi\right) * \alpha K\right\|_{2} \\
\leqq & \|f\|_{2}\left\|D\left(w_{j} D_{j} \phi\right)\right\|_{2}\|\alpha K\|_{1} \\
\leqq & C\|f\|_{2}\left(\|D w\|_{2}\|D \phi\|_{\infty}+\|w\|_{2}\left\|D^{2} \phi\right\|_{\infty}\right) \varepsilon^{2} .
\end{aligned}
$$

We use Definition 3.3, $\operatorname{div}(w)=0, \operatorname{spt}(\phi) \subset B(a, r), \operatorname{spt}(\beta) \subset B(0,2 r)$, and the generalized Hölder inequality to estimate

$$
\begin{aligned}
& \left|\int f_{i} D_{i}(\operatorname{div}(w \phi) * \beta K)\right| \\
= & \left|\int\left(w_{j} * H_{\zeta}\right) D_{j} w_{i} D_{i}\left(\left(w_{k} D_{k} \phi\right) * \beta K\right)\right| \\
= & \left|\int\left(w_{j} * H_{\zeta}\right) w_{i} D_{i j}\left(\left(w_{k} D_{k} \phi\right) * \beta K\right)\right| \\
= & \left|\int_{B(a, 3 r)}\left(w_{j} * H_{\zeta}\right) w_{i} D_{i j}\left(\left(w_{k} D_{k} \phi\right) * \beta K\right)\right| \\
\leqq & \sum_{i, j}\left(\int_{B(a, 3 r)}\left|w * H_{\zeta}\right|^{3}\right)^{1 / 3}\left(\int_{B(a, 3 r)}|w|^{3}\right)^{1 / 3}\left\|D_{i j}\left(\left(w_{k} D_{k} \phi\right) * \beta K\right)\right\|_{3} .
\end{aligned}
$$

From Lemma 3.2 we obtain

$$
\begin{aligned}
\int_{B(a, 3 r)}\left|w * H_{\zeta}\right|^{3} & \leqq C r^{5}\left(\int\left|\left(w * H_{\zeta}\right)(x)\right|^{3}(|x-a|+r)^{-5} d x\right) \\
& \leqq C r^{5}\left(\int|w(x)|^{3}(|x-a|+r)^{-5} d x\right) .
\end{aligned}
$$

We also have

$$
\int_{B(a, 3 r)}|w|^{3} \leqq C r^{5}\left(\int|w(x)|^{3}(|x-a|+r)^{-5} d x\right) .
$$

Now [7, Proposition 3, p. 59], $\operatorname{spt}(\phi) \subset B(a, r), \operatorname{spt}(\beta) \subset B(0,2 r)$, Young's inequality, and (3.9) yield

$$
\begin{aligned}
& \left\|D_{i j}\left(\left(w_{k} D_{k} \phi\right) * \beta K\right)\right\|_{3} \leqq C\left\|\Delta\left(\left(w_{k} D_{k} \phi\right) * \beta K\right)\right\|_{3}=C\left\|_{w_{k}} D_{k} \phi * \Delta(\beta K)\right\|_{3} \\
& \leqq C\left\|_{w_{k}} D_{k} \phi\right\|_{3}\|\Delta(\beta K)\|_{1} \leqq C\left\|_{w_{k}} D_{k} \phi\right\|_{3} \leqq C\left(\int_{B(a, r)}|w|^{3}\right)^{1 / 3}\|D \phi\|_{\infty} \\
& \leqq C r^{5 / 3}\|D \phi\|_{\infty}\left(\int|w(x)|^{3}(|x-a|+r)^{-5} d x\right)^{1 / 3} .
\end{aligned}
$$

Combining (3.11), (3.12), (3.13), and (3.14) we obtain

$$
\left|\int f_{i} D_{i}(\operatorname{div}(w \phi) * \beta K)\right| \leqq C r^{5}\|D \phi\|_{\infty}\left(\int|w(x)|^{3}(|x-a|+r)^{-5} d x\right) .
$$


From Definition 3.3 and the hypothesis $\operatorname{div}(w)=0$ we obtain

$$
\begin{aligned}
& \left|\int f_{i} D_{i}(\operatorname{div}(w \phi) * \gamma K)\right| \\
= & \left|\int\left(w_{j} * H_{\zeta}\right) D_{j} w_{i} D_{i}\left(D_{k}\left(w_{k} \phi\right) * \gamma K\right)\right| \\
= & \left|\int\left(w_{j} * H_{\zeta}\right) w_{i} D_{i j k}\left(w_{k} \phi * \gamma K\right)\right| \\
= & \left|\int\left(w_{j} * H_{\zeta}\right) w_{i}\left(w_{k} \phi * D_{i j k}(\gamma K)\right)\right| .
\end{aligned}
$$

Now (3.8), $\operatorname{spt}(\phi) \subset B(a, r)$, and Hölder's inequality yield

$$
\begin{aligned}
& \left|\left(w_{k} \phi * D_{i j k}(\gamma K)\right)(x)\right| \\
= & \left|\int\left(w_{k} \phi\right)(y)\left(D_{i j k}(\gamma K)\right)(x-y) d y\right| \\
\leqq & C\left(\int|(w \phi)(y)|(|x-y|+r)^{-5} d y\right) \\
\leqq & C\left(\int|(w \phi)(y)| d y\right)\left(\sup \left\{(|x-y|+r)^{-5}: y \in B(a, r)\right\}\right) \\
\leqq & C\|\phi\|_{\infty}\left(\int_{B(a, r)}|w(y)| d y\right)(|x-a|+r)^{-5} \\
\leqq & C \phi \|_{\infty}\left(\int_{B(a, r)}|w(y)|^{3} d y\right)^{1 / 3}\left(\int_{B(a, r)}(1)^{3 / 2}\right)^{2 / 3}(|x-a|+r)^{-5} \\
\leqq & \left.C\left\|_{\infty}\right\|_{\infty} \int_{B(a, r)}|w(y)|^{3} d y\right)^{1 / 3} r^{8 / 3}(|x-a|+r)^{-5} \\
\leqq & C\left\|_{\phi}\right\|_{\infty} r^{13 / 3}\left(\int|w(y)|^{3}(|y-a|+r)^{-5} d y\right)^{1 / 3}(|x-a|+r)^{-5} .
\end{aligned}
$$

Combining (3.16), (3.17), and (3.6) we obtain

$$
\begin{aligned}
& \left|\int f_{i} D_{i}(\operatorname{div}(w \phi) * \gamma K)\right| \\
\leqq & C\|\phi\|_{\infty} r^{13 / 3}\left(\int|w(y)|^{3} J(y) d y\right)^{1 / 3}\left(\int\left|\left(w * H_{\zeta}\right)(x)\right||w(x)| J(x) d x\right) .
\end{aligned}
$$

Furthermore, the generalized Hölder inequality, Lemma 3.2, and (3.6) yield

$$
\begin{aligned}
& \int\left|\left(w * H_{\zeta}\right)(x)\right||w(x)| J(x) d x \\
= & \int\left(\left|\left(w * H_{\zeta}\right)(x)\right|(J(x))^{1 / 3}\right)\left(|w(x)|(J(x))^{1 / 3}\right)\left((J(x))^{1 / 3}\right) d x \\
\leqq & \left(\int\left|\left(w * H_{\zeta}\right)(x)\right|^{3} J(x) d x\right)^{1 / 3}\left(\int|w(x)|^{3} J(x) d x\right)^{1 / 3}\left(\int J(x) d x\right)^{1 / 3} \\
\leqq & C\left(\int|w(x)|^{3} J(x) d x\right)^{2 / 3}\left(\int J(x) d x\right)^{1 / 3} \\
\leqq & C r^{-1 / 3}\left(\int|w(x)|^{3} J(x) d x\right)^{2 / 3} .
\end{aligned}
$$

Combining (3.18), (3.19), and (3.6) we obtain

$$
\left|\int f_{i} D_{i}(\operatorname{div}(w \phi) * \gamma K)\right| \leqq C\left\|_{\phi}\right\|_{\infty} r^{4}\left(\int|w(x)|^{3}(|x-a|+r)^{-5} d x\right) .
$$

We use $\operatorname{div}(w)=0$, the Schwarz inequality, Definition 3.3, (3.8), and Young's inequality to estimate

$$
\begin{aligned}
& \left|\int f_{i} D_{i}(\operatorname{div}(w \phi) * \delta K)\right| \\
= & \left|\int f_{i}\left(w_{k} D_{k} \phi * D_{i}(\delta K)\right)\right| \\
\leqq & \|f\|_{1}\left\|w_{k} D_{k} \phi * D(\delta K)\right\|_{\infty} \\
\leqq & \|f\|_{1}\left\|w_{k} D_{k} \phi\right\|_{2}\|D(\delta K)\|_{2} \\
\leqq & C\left\|_{w * H_{\zeta}}\right\|_{2}\|D w\|_{2}\left\|_{w}\right\|_{2}\|D \phi\|_{\infty} \varepsilon \\
\leqq & C\left\|_{w}\right\|_{2}\left\|H_{\zeta}\right\|_{1}\|D w\|_{2}\|w\|_{2}\|D \phi\|_{\infty} \varepsilon .
\end{aligned}
$$


Now we use (3.7), (3.10), (3.15), (3.20), (3.21), (3.3), $\|w\|_{2}<\infty,\|D w\|_{2}<\infty$, $\left\|H_{\zeta}\right\|_{1}<\infty, \phi \in C_{0}^{\infty}\left(R^{4}, R\right)$, and the fact that $\varepsilon$ can be made arbitrarily small to conclude

$$
\begin{aligned}
& \left|\int f_{i} D_{i}(\operatorname{div}(w \phi) * K)\right| \\
\leqq & C\left(\|D \phi\|_{\infty}+r^{-1}\|\phi\|_{\infty}\right) r^{5}\left(\int|w(x)|^{3}(|x-a|+r)^{-5} d x\right) .
\end{aligned}
$$

Using Definition 3.3, div. $(w)=0, \operatorname{spt}(\phi) \subset B(a, r)$, Hölder's inequality, (3.12), and (3.6) we find

$$
\begin{aligned}
& \left|\int f_{i} w_{i} \phi\right|=\left|\int\left(w_{j} * H_{\zeta}\right)\left(D_{j} w_{i}\right) w_{i} \phi\right|=(1 / 2)\left|\int\left(w_{j} * H_{\zeta}\right) D_{j}\left(|w|^{2}\right) \phi\right| \\
= & \left.(1 / 2)\left|\int\left(w_{j} * H_{\zeta}\right)\right| w\right|^{2} D_{j} \phi \mid \leqq(1 / 2)\left(\int_{B(a, r)}\left|w * H_{\zeta}\right||w|^{2}\right)\|D \phi\|_{\infty} \\
\leqq & (1 / 2)\|D \phi\|_{\infty}\left(\int_{B(a, r)}\left|w * H_{\zeta}\right|^{3}\right)^{1 / 3}\left(\int_{B(a, r)}|w|^{3}\right)^{2 / 3} \\
\leqq & C\|D \phi\|_{\infty} r^{5 / 3}\left(\int|w(x)|^{3} J(x) d x\right)^{1 / 3} r^{10 / 3}\left(\int|w(x)|^{3} J(x) d x\right)^{2 / 3} \\
= & C\|D \phi\|_{\infty} r^{5}\left(\int|w(x)|^{3}(|x-a|+r)^{-5} d x\right) .
\end{aligned}
$$

Finally (3.5) (with $\phi$ replaced by $w \phi$ ), (3.22), and (3.23) imply the conclusion of Lemma 3.4.

For every $n \in\{1,2,3, \ldots\}$ we set $r_{n}=n \zeta^{1 / 2}$ and construct a $C^{\infty}$ function $\phi_{n}: R^{4} \rightarrow[0,1]$ such that $\phi_{n}(x)=1$ if $x \in B\left(0, r_{n} / 2\right), \phi_{n}(x)=0$ if $x \notin B\left(0, r_{n}\right)$, and $\left\|D \phi_{n}\right\|_{\infty} \leqq C r_{n}^{-1}$. Lemma 3.4 (with $a=0$ ) yields

$$
\left|\int g_{i} w_{i} \phi_{n}\right| \leqq C r_{n}^{-1}\left\|_{w}\right\|_{3}^{3} \text {. }
$$

From Lemma 3.1, $\|w\|_{2}<\infty$, and $\|D w\|_{2}<\infty$ we conclude $\|w\|_{3}<\infty$. We also have $g \in L^{2}\left(R^{4}, R^{4}\right)$ (see Definition 3.3) and $w \in L^{2}\left(R^{4}, R^{4}\right)$. Hence we can take the limit as $n \rightarrow \infty$ in (3.24) and conclude

$$
\int g_{i} w_{i}=0 \text {. }
$$

Lemma 3.5. Suppose $a \in R^{4}, p$ is an integer, $t$ is a real number, $\zeta \leqq t \leqq 2^{-2 p+1}$, $\alpha: R^{4} \rightarrow[0,1]$ is a $C^{\infty}$ function, $\alpha(x)=1$ if $x \in B\left(a, 2^{-p-1}\right), \alpha(x)=0$ if $x \notin B\left(a, 2^{-p}\right)$, and $\|D \alpha\|_{\infty} \leqq 2^{p+2}$. Then

$$
\left|\int g_{i}(x) w_{i}(x) H_{t}(x-a) \alpha(x) d x\right| \leqq C\left(\int|w(x)|^{3}\left(|x-a|+t^{1 / 2}\right)^{-5} d x\right) .
$$

Proof. We define $k$ by the properties

$$
2^{-2 k-1}<t \leqq 2^{-2 k+1}, k \text { is an integer. }
$$

The hypotheses imply $p \leqq k$. For every integer $j$ satisfying $p \leqq j \leqq k$ we construct $\alpha_{j}$ as follows: We set $\alpha_{p}=\alpha$; if $p<j \leqq k$ we choose a $C^{\infty}$ function $\alpha_{j}: R^{4} \rightarrow[0,1]$ such that $\alpha_{j}(x)=1$ if $x \in B\left(a, 2^{-j-1}\right), \alpha_{j}(x)=0$ if $x \notin B\left(a, 2^{-j}\right),\left\|D \alpha_{j}\right\|_{\infty} \leqq 2^{j+2}$. We define $\phi_{j}: R^{4} \rightarrow R$ as follows :

$$
\begin{aligned}
& \phi_{j}(x)=H_{t}(x-a)\left(\alpha_{j}(x)-\alpha_{j+1}(x)\right) \text { if } \quad p \leqq j<k \\
& \phi_{k}(x)=H_{t}(x-a) \alpha_{k}(x) .
\end{aligned}
$$


From (3.26) and the hypotheses we conclude $2^{-j+1} \geqq 2^{-k+1} \geqq(2 t)^{1 / 2}>\zeta^{1 / 2}$ if $p \leqq j \leqq k$. We also have $\operatorname{spt}\left(\phi_{j}\right) \subset B\left(a, 2^{-j+1}\right)$. The last two statements and Lemma 3.4 yield

$$
\begin{aligned}
& \left|\int g_{i}(x) w_{i}(x) H_{t}(x-a) \alpha(x) d x\right| \\
\leqq & \sum_{j=p}^{k}\left|\int g_{i}(x) w_{i}(x) \phi_{j}(x) d x\right| \\
\leqq & \sum_{j=p}^{k} C\left(\left\|D \phi_{j}\right\|_{\infty}+2^{j-1}\left\|\phi_{j}\right\|_{\infty}\right) 2^{-5 j+5}\left(\int|w(x)|^{3}\left(|x-a|+2^{-j+1}\right)^{-5} d x\right) .
\end{aligned}
$$

We have

$$
0<H_{t}(x) \leqq C t^{1 / 2}\left(|x|+t^{1 / 2}\right)^{-5},\left|D\left(H_{t}\right)(x)\right| \leqq C t^{1 / 2}\left(|x|+t^{1 / 2}\right)^{-6} .
$$

We also have

$$
\begin{aligned}
& \operatorname{spt}\left(\alpha_{j}-\alpha_{j+1}\right) \subset B\left(a, 2^{-j}\right)-B\left(a, 2^{-j-2}\right) \text { if } j<k, \\
& \operatorname{spt}\left(\alpha_{k}\right) \subset B\left(a, 2^{-k}\right) .
\end{aligned}
$$

From (3.26), (3.28), and (3.29) we conclude

$$
\begin{aligned}
& 0<H_{t}(x-a) \leqq C 2^{5 j-k} \quad \text { if } \quad x \in \operatorname{spt}\left(\alpha_{j}-\alpha_{j+1}\right) \quad \text { and } j<k, \\
& 0<H_{t}(x-a) \leqq C 2^{4 k} \quad \text { if } \quad x \in \operatorname{spt}\left(\alpha_{k}\right), \\
& \left|D\left(H_{t}\right)(x-a)\right| \leqq C 2^{6 j-k} \quad \text { if } \quad x \in \operatorname{spt}\left(\alpha_{j}-\alpha_{j+1}\right) \text { and } j<k, \\
& \left|D\left(H_{t}\right)(x-a)\right| \leqq C 2^{5 k} \quad \text { if } \quad x \in \operatorname{spt}\left(\alpha_{k}\right) .
\end{aligned}
$$

From (3.30) and the properties of $\alpha_{j}$ we obtain

$$
\left\|D \phi_{j}\right\|_{\infty}+2^{j-1}\left\|\phi_{j}\right\|_{\infty} \leqq C 2^{6 j-k} \text { if } p \leqq j \leqq k .
$$

Now (3.27), (3.31), and (3.26) yield

$$
\begin{aligned}
& \left|\int g_{i}(x) w_{i}(x) H_{t}(x-a) \alpha(x) d x\right| \\
\leqq & \sum_{j=p}^{k} C 2^{j-k}\left(\int|w(x)|^{3}\left(|x-a|+2^{-j+1}\right)^{-5} d x\right) \\
\leqq & \sum_{j=p}^{k} C 2^{j-k}\left(\int|w(x)|^{3}\left(|x-a|+2^{-k}\right)^{-5} d x\right) \\
\leqq & C\left(\int|w(x)|^{3}\left(|x-a|+2^{-k}\right)^{-5} d x\right) \\
\leqq & C\left(\int|w(x)|^{3}\left(|x-a|+t^{1 / 2}\right)^{-5} d x\right) .
\end{aligned}
$$

The lemma has been proved.

Lemma 3.6. If $0<t<\infty, f \in C^{\infty}\left(R^{4}, R\right), \int|f|^{2}<\infty$, and $\int|D f|^{2}<\infty$ then

$$
\left\|f-\left(f * H_{t}\right)\right\|_{2} \leqq C t^{1 / 2}\|D f\|_{2} .
$$

Proof. Define $g: R^{4} \times(0, \infty) \rightarrow R$ by $g(x, s)=\left(f * H_{s}\right)(x)$. The relation $D_{t} g=\Delta g$ yields

$$
\begin{aligned}
\left(f * H_{t}\right)(x)-f(x) & =\int_{0}^{t} D_{t} g(x, s) d s=\int_{0}^{t} \Delta g(x, s) d s \\
& =\int_{0}^{t} D_{i i}\left(f * H_{s}\right)(x) d s=\int_{0}^{t}\left(D_{i} f * D_{i} H_{s}\right)(x) d s .
\end{aligned}
$$


Hence Minkowski's integral inequality and Young's inequality yield

$$
\begin{aligned}
& \left(\int\left|\left(f * H_{t}\right)(x)-f(x)\right|^{2} d x\right)^{1 / 2} \\
= & \left(\left.\int\left|\int_{0}^{t}\left(D_{i} f * D_{i} H_{s}\right)(x) d s\right|^{2} d x\right|^{1 / 2}\right. \\
\leqq & \int_{0}^{t}\left(\int\left|\left(D_{i} f * D_{i} H_{s}\right)(x)\right|^{2} d x\right)^{1 / 2} d s \\
\leqq & \int_{0}^{t}\|D f\|_{2}\left\|D H_{s}\right\|_{1} d s=\|D f\|_{2}\left(\int_{0}^{t}\left(C s^{-1 / 2}\right) d s\right)=C\|D f\|_{2} t^{1 / 2} .
\end{aligned}
$$

\section{Section 4. Estimates on Approximate Solutions}

Throughout this section we fix positive real numbers $\zeta$ and $d$ such that (see Section 1)

$$
d \leqq \zeta, d L\left\|H_{\zeta}\right\|_{2}^{2} \leqq 1 .
$$

Definition 4.1. We use induction to define functions $v^{-1}, v^{0}, v^{1}, v^{2}, \ldots$ such that $v^{k} \in L^{2}\left(R^{4}, R^{4}\right)$ and

$$
\left\|v^{k}\right\|_{2}^{2} \leqq L, \operatorname{div}\left(v^{k}\right)=0 .
$$

We set $v^{-1}=v$ (see Section 1). Suppose that $k \geqq 0$ and $v^{k-1} \in L^{2}\left(R^{4}, R^{4}\right)$ has been defined so that (4.3) holds :

$$
\left\|v^{k-1}\right\|_{2}^{2} \leqq L, \operatorname{div}\left(v^{k-1}\right)=0 .
$$

We will define $v^{k}$ with the aid of several auxiliary functions. Let $u^{k}: R^{4} \times[k d, \infty) \rightarrow R^{4}$ be given by $u^{k}(x, k d)=v^{k-1}(x), u^{k}(x, k d+t)=\left(v^{k-1} * H_{t}\right)(x)$ if $t>0$. Let $w^{k}: R^{4} \rightarrow R^{4}$ be given by $w^{k}(x)=u^{k}(x, k d+d)$. The relationship $D_{t}\left(u^{k}\right)=\Delta\left(u^{k}\right)$ implies

$$
\left\|w^{k}\right\|_{2}^{2}=\left\|v^{k-1}\right\|_{2}^{2}-2\left(\int_{k d}^{k d+d} \int\left|D u^{k}(x, t)\right|^{2} d x d t\right) .
$$

Similarly, the relationship $D_{t}\left(D_{i} u^{k}\right)=\Delta\left(D_{i} u^{k}\right)$ implies

$$
\left\|D_{i} w^{k}\right\|_{2}^{2}=\int\left|D_{i} u^{k}(x, t)\right|^{2} d x-2\left(\int_{t}^{k d+d} \int\left|D\left(D_{i} u^{k}\right)(x, s)\right|^{2} d x d s\right)
$$

for all $t \in(k d, k d+d)$. Averaging (4.5) over $t$ and summing over $i$ we obtain

$$
\left\|D w^{k}\right\|_{2}^{2} \leqq d^{-1}\left(\int_{k d}^{k d+d} \int\left|D u^{k}(x, t)\right|^{2} d x d t\right) .
$$

From (4.3), (4.4), (4.6), and the definition of $w^{k}$ we conclude that $w^{k}$ satisfies

$$
\begin{aligned}
& \left\|w^{k}\right\|_{2}^{2} \leqq\left\|v^{k-1}\right\|_{2}^{2} \leqq L, \\
& \left\|D w^{k}\right\|_{2}^{2} \leqq(2 d)^{-1}\left\|v^{k-1}\right\|_{2}^{2} \leqq(2 d)^{-1} L, \\
& w^{k} \in C^{\infty}\left(R^{4}, R^{4}\right) \text { and } \operatorname{div}\left(w^{k}\right)=0 .
\end{aligned}
$$


Hence we can replace $w$ by $w^{k}$ in Section 3 and construct functions $f^{k}$ and $g^{k}$ corresponding to $f$ and $g$ in Definition 3.3. We set

$$
v^{k}=w^{k}-(d)\left(g^{k}\right) \text {. }
$$

In order to complete the inductive definition, we must show that (4.2) holds. Using (3.4), (3.3), (4.7), and (4.6) we obtain

$$
\begin{aligned}
d^{2}\left\|g^{k}\right\|_{2}^{2} & \leqq d^{2}\left\|f^{k}\right\|_{2}^{2} \leqq d^{2}\left\|w^{k}\right\|_{2}^{2}\left\|H_{\zeta}\right\|_{2}^{2}\left\|D w^{k}\right\|_{2}^{2} \leqq d^{2} L\left\|H_{\zeta}\right\|_{2}^{2}\left\|D w^{k}\right\|_{2}^{2} \\
& \leqq d L\left\|H_{\zeta}\right\|_{2}^{2}\left(\int_{k d}^{k d+d} \int\left|D u^{k}(x, t)\right|^{2} d x d t\right) .
\end{aligned}
$$

From (4.10), (3.25), (4.4), (4.11), and (4.1) we obtain

$$
\begin{aligned}
\left\|v^{k}\right\|_{2}^{2} & =\left\|w^{k}\right\|_{2}^{2}+d^{2}\left\|g^{k}\right\|_{2}^{2} \\
& =\left\|v^{k-1}\right\|_{2}^{2}-2\left(\int_{k d}^{k d+d} \int\left|D u^{k}(x, t)\right|^{2} d x d t\right)+d^{2}\left\|g^{k}\right\|_{2}^{2} \\
& \leqq\left\|v^{k-1}\right\|_{2}^{2}-\int_{k d}^{k d+d} \int\left|D u^{k}(x, t)\right|^{2} d x d t .
\end{aligned}
$$

From (4.12) and (4.3) we conclude

$$
\left\|v^{k}\right\|_{2}^{2} \leqq\left\|v^{k-1}\right\|_{2}^{2} \leqq L \text {. }
$$

If $\phi \in C_{0}^{\infty}\left(R^{4}, R\right)$ then $(\nabla \operatorname{div}(\nabla \phi)) * K=\nabla(\Delta \phi * K)=\nabla \phi$. Hence (3.5) yields $\int g^{k} \cdot \nabla \phi$ $=0$. We conclude $\operatorname{div}\left(g^{k}\right)=0$. Combining this with (4.9), (4.10), and (4.13) we obtain that (4.2) holds. The definition of the $v^{k}$ is complete.

We define $u: R^{4} \times[0, \infty) \rightarrow R^{4}$ (this is not the $u$ in Theorem 1.1) by

$$
u(x, t)=u^{k}(x, t) \quad \text { if } \quad k d \leqq t<k d+d .
$$

From (4.2) and (4.12) we conclude

$$
\int_{0}^{\infty} \int|D u(x, t)|^{2} d x d t \leqq L
$$

If $t \geqq k d$ then the property $\int\left|u^{k}(x, t)\right|^{2} d x \leqq\left\|v^{k-1}\right\|_{2}^{2}$ follows in the same way as (4.4). Hence (4.2) yields

$$
\int|u(x, t)|^{2} d x \leqq L \quad \text { for all } t \geqq 0 .
$$

The argument in the proof of Lemma 3.1, (4.15), and (4.16) yield

$$
\begin{aligned}
& \int_{0}^{\infty} \int|u(x, t)|^{3} d x d t \\
& \quad \leqq C\left(\int_{0}^{\infty}\left(\int|D u(x, t)|^{2} d x\right)\left(\int|u(x, t)|^{2} d x\right)^{1 / 2} d t\right) \leqq C L^{3 / 2} .
\end{aligned}
$$

Definition 4.2. In Lemmas 4.3 and 4.4 we fix $a \in R^{4}, 0<b<\infty$, and integers $p$ and $q$ satisfying $p<q, 2^{-2 p} \leqq b$, and $d \leqq 2^{-2 p-2}$. We construct $C^{\infty}$ functions $\alpha: R^{4} \rightarrow[0,1]$ 
and $\beta: R \rightarrow[0,1]$ such that $\alpha(x)=1$ if $x \in B\left(a, 2^{-p-1}\right), \alpha(x)=0$ if $x \notin B\left(a, 2^{-p}\right),\|D \alpha\|_{\infty}$ $\leqq 2^{p+2},\left\|D^{2} \alpha\right\|_{\infty} \leqq C 2^{2 p}, \beta(t)=1$ if $t \geqq b-2^{-2 p-2}, \beta(t)=0$ if $t \leqq b-2^{-2 p}+d$, and $\|(\partial / \partial t) \beta\|_{\infty} \leqq C 2^{2 p}$. We define $\phi: R^{4} \times[0, b] \rightarrow R$ by $[$ see (1.4)]

$$
\phi(x, t)=\left(H\left[b-t+2^{-2 q}\right]\right)(x-a) \alpha(x) \beta(t) .
$$

We have $\left(D_{t} \phi+\Delta \phi\right)(x, t)=0$ if $|x-a|<2^{-p-1}$ and $b-2^{-2 p-2}<t<b$. From this we conclude

$$
\left\|D_{t} \phi+\Delta \phi\right\|_{\infty} \leqq C 2^{6 p} .
$$

We also have

$$
\|\phi\|_{\infty} \leqq C 2^{4 q} \text {. }
$$

Lemma 4.3. If $k \in\{0,1,2, \ldots\}, b-2^{-2 p} \leqq k d+d \leqq b$ and $\zeta \leqq 2^{-2 q}$ (see Definition 4.2) then

$$
\begin{aligned}
& \int\left|u^{k+1}(x, k d+d)\right|^{2} \phi(x, k d+d) d x \\
& \quad-\int\left|u^{k}(x, k d+d)\right|^{2} \phi(x, k d+d) d x \\
& \quad \leqq C\left(\int_{k d}^{k d+d} \int|u(x, t)|^{3}\left(|x-a|+\left(b-t+2^{-2 q}\right)^{1 / 2}\right)^{-5} d x d t\right) \\
& \quad+C d L\left\|H_{\zeta}\right\|{ }_{2}^{2}\left(2^{4 q}\right)\left(\int_{k d}^{k d+d} \int|D u(x, t)|^{2} d x d t\right) .
\end{aligned}
$$

Proof. From Definition 4.1 and (4.10) we obtain

$$
\begin{aligned}
\int\left|u^{k+1}(x, k d+d)\right|^{2} \phi(x, k d+d) d x & \\
& \quad-\int\left|u^{k}(x, k d+d)\right|^{2} \phi(x, k d+d) d x \\
= & \int\left|v^{k}(x)\right|^{2} \phi(x, k d+d) d x-\int\left|w^{k}(x)\right|^{2} \phi(x, k d+d) d x \\
= & -2 d\left(\int g_{i}^{k}(x) w_{i}^{k}(x) \phi(x, k d+d) d x\right)+d^{2}\left(\int\left|g^{k}(x)\right|^{2} \phi(x, k d+d) d x\right) .
\end{aligned}
$$

Using (4.20), (4.11), and (4.14) we find

$$
\begin{aligned}
& d^{2}\left(\int\left|g^{k}(x)\right|^{2} \phi(x, k d+d) d x\right) \leqq C d^{2}\left\|g^{k}\right\|_{2}^{2}\left(2^{4 q}\right) \\
& \quad \leqq C d L\left\|H_{\zeta}\right\|_{2}^{2}\left(2^{4 q}\right)\left(\int_{k d}^{k d+d} \int|D u(x, t)|^{2} d x d t\right) .
\end{aligned}
$$

The hypotheses on $p, q$, and $k$ imply

$$
b-(k d+d)+2^{-2 q} \leqq 2^{-2 p}+2^{-2 q}<2^{-2 p+1} .
$$

The hypothesis of Lemma 4.3 implies

$$
\zeta \leqq 2^{-2 q} \leqq b-(k d+d)+2^{-2 q} .
$$

Now (4.18), Definition 4.2, (4.23), (4.24), and Lemma 3.5 yield [see (1.4)]

$$
\begin{aligned}
& \left|\int g_{i}^{k}(x) w_{i}^{k}(x) \phi(x, k d+d) d x\right| \\
& \quad=\beta(k d+d)\left|\int g_{i}^{k}(x) w_{i}^{k}(x)\left(H\left[b-(k d+d)+2^{-2 q}\right]\right)(x-a) \alpha(x) d x\right| \\
& \quad \leqq C\left(\int\left|w^{k}(x)\right|^{3}\left(|x-a|+\left(b-(k d+d)+2^{-2 q}\right)^{1 / 2}\right)^{-5} d x\right) .
\end{aligned}
$$


For every $t \in(k d, k d+d)$ we define $h^{t}: R^{4} \rightarrow R^{4}$ by

$$
h^{t}(x)=u^{k}(x, t)=u(x, t) .
$$

From Definition 4.1 and the semigroup property $H_{a} * H_{b}=H_{a+b}$ (see $[8$, Corollary 1.28, p. 16]) we obtain

$$
\begin{aligned}
w^{k}(x) & =u^{k}(x, k d+d)=\left(v^{k-1} * H_{d}\right)(x) \\
& =\left(v^{k-1} * H[t-k d] * H[k d+d-t]\right)(x) \\
& =\left(h^{t} * H[k d+d-t]\right)(x) .
\end{aligned}
$$

The hypotheses of Lemma 4.3 and (4.1) yield the following for $k d<t<k d+d$ :

$$
(k d+d-t)^{1 / 2}<d^{1 / 2} \leqq \zeta^{1 / 2} \leqq\left(2^{-2 q}\right)^{1 / 2} \leqq\left(b-(k d+d)+2^{-2 q}\right)^{1 / 2} .
$$

Now (4.27), (4.28), and the proof of Lemma 3.2 yield

$$
\begin{aligned}
& \int\left|w^{k}(x)\right|^{3}\left(|x-a|+\left(b-(k d+d)+2^{-2 q}\right)^{1 / 2}\right)^{-5} d x \\
& \quad \leqq C\left(\int\left|h^{t}(x)\right|^{3}\left(|x-a|+\left(b-(k d+d)+2^{-2 q}\right)^{1 / 2}\right)^{-5} d x\right)
\end{aligned}
$$

if $k d<t<k d+d$. From (4.1) and the hypotheses $\zeta \leqq 2^{-2 q}, k d+d \leqq b$ we obtain $d \leqq \zeta$ $\leqq 2^{-2 q}$ and $d \leqq b-k d$. This implies $d \leqq(1 / 2)\left(b-k d+2^{-2 q}\right)$, which in turn implies

$$
\begin{aligned}
& b-(k d+d)+2^{-2 q}=\left(b-k d+2^{-2 q}\right)-d \geqq(1 / 2)\left(b-k d+2^{-2 q}\right) \\
& \quad \geqq(1 / 2)\left(b-t+2^{-2 q}\right)
\end{aligned}
$$

whenever $k d<t<k d+d$. We conclude

$$
\left(b-t+2^{-2 q}\right)^{1 / 2}>\left(b-(k d+d)+2^{-2 q}\right)^{1 / 2} \geqq(1 / 2)^{1 / 2}\left(b-t+2^{-2 q}\right)^{1 / 2}
$$

if $k d<t<k d+d$. Now (4.30) implies

$$
\begin{aligned}
& \int\left|h^{t}(x)\right|^{3}\left(|x-a|+\left(b-(k d+d)+2^{-2 q}\right)^{1 / 2}\right)^{-5} d x \\
\leqq & C\left(\int\left|h^{t}(x)\right|^{3}\left(|x-a|+\left(b-t+2^{-2 q}\right)^{1 / 2}\right)^{-5} d x\right)
\end{aligned}
$$

if $k d<t<k d+d$. Using (4.29) and (4.31), averaging over $t$, and using (4.26) we obtain

$$
\begin{aligned}
& \int\left|w^{k}(x)\right|^{3}\left(|x-a|+\left(b-(k d+d)+2^{-2 q}\right)^{1 / 2}\right)^{-5} d x \\
\leqq & C d^{-1}\left(\int_{k d}^{k d+d} \int|u(x, t)|^{3}\left(|x-a|+\left(b-t+2^{-2 q}\right)^{1 / 2}\right)^{-5} d x d t\right) .
\end{aligned}
$$

Finally, (4.21), (4.22), (4.25), and (4.32) yield the conclusion of the lemma.

Lemma 4.4. If $b-2^{-2 q} \leqq s \leqq b$ and $\zeta \leqq 2^{-2 q}$ (see Definition 4.2) then

$$
\begin{aligned}
& (1 / 2)\left(\int|u(x, s)|^{2} \phi(x, s) d x\right)+\int_{b-2^{-2 p}}^{s} \int|D u(x, t)|^{2} \phi(x, t) d x d t \\
\leqq & C\left(\int_{b-2-2 p}^{b} \int|u(x, t)|^{3}\left(|x-a|+\left(b-t+2^{-2 q}\right)^{1 / 2}\right)^{-5} d x d t\right) \\
+ & C d L^{2}\left\|H_{\zeta}\right\|{ }_{2}^{2}\left(2^{4 q}\right)+C\left(2^{6 p}\right)\left(\int_{b-2^{-2 p}}^{b} \int_{B\left(a, 2^{-p}\right)}|u(x, t)|^{2} d x d t\right) .
\end{aligned}
$$


Proof. We define integers $k^{\prime}$ and $k^{\prime \prime}$ by the relations

$$
\left(k^{\prime}-1\right) d<b-2^{-2 p} \leqq k^{\prime} d, k^{\prime \prime} d+d \leqq s<\left(k^{\prime \prime}+1\right) d+d .
$$

From (4.33) and $b-2^{-2 p} \geqq 0$ we obtain $k^{\prime} \geqq 0$. From Definition 4.2 we obtain $2^{-2 p}-2^{-2 q} \geqq(3 / 4) 2^{-2 p} \geqq 3 d$. Hence (4.33) and the hypotheses yield

$$
k^{\prime} d<d+b-2^{-2 p} \leqq-2 d+b-2^{-2 q} \leqq-2 d+s \leqq k^{\prime \prime} d .
$$

Since $k^{\prime} \geqq 0$ we conclude $0 \leqq k^{\prime}<k^{\prime \prime}$. From (4.14) we obtain

$$
\begin{aligned}
& (1 / 2)\left(\int\left|u\left(x, k^{\prime \prime} d+d\right)\right|^{2} \phi\left(x, k^{\prime \prime} d+d\right) d x\right) \\
& -(1 / 2)\left(\int\left|u\left(x, k^{\prime} d\right)\right|^{2} \phi\left(x, k^{\prime} d\right) d x\right) \\
= & (1 / 2)\left(\sum_{k=k^{\prime}}^{k^{\prime \prime}}\left(\int|u(x, k d+d)|^{2} \phi(x, k d+d) d x-\int|u(x, k d)|^{2} \phi(x, k d) d x\right)\right) \\
= & (1 / 2)\left(\sum_{k=k^{\prime}}^{k^{\prime \prime}}\left(\int\left|u^{k+1}(x, k d+d)\right|^{2} \phi(x, k d+d) d x-\int\left|u^{k}(x, k d)\right|^{2} \phi(x, k d) d x\right)\right) \\
= & (1 / 2)\left(\sum _ { k = k ^ { \prime } } ^ { k ^ { \prime \prime } } \left(\int\left|u^{k+1}(x, k d+d)\right|^{2} \phi(x, k d+d) d x\right.\right. \\
& +(1 / 2)\left(\sum _ { k = k ^ { \prime } } ^ { k ^ { \prime \prime } } \left(\int\left|u^{k}(x, k d+d)\right|^{2} \phi(x, k d+d) d x\right.\right. \\
& \left.\left.-\int\left|u^{k}(x, k d)\right|^{2} \phi(x, k d) d x\right)\right) .
\end{aligned}
$$

Taking the inner product of the relation $D_{t}\left(u^{k}\right)=\Delta\left(u^{k}\right)$ with $u^{k} \phi$ (see Definition 4.1) and using (4.14), Definition 4.2, and (4.19) we obtain the following whenever $k^{\prime} \leqq k \leqq k^{\prime \prime}$ :

$$
\begin{aligned}
& (1 / 2)\left(\int\left|u^{k}(x, k d+d)\right|^{2} \phi(x, k d+d) d x\right) \\
& -(1 / 2)\left(\int\left|u^{k}(x, k d)\right|^{2} \phi(x, k d) d x\right) \\
= & -\int_{k d}^{k d+d} \int\left|D u^{k}(x, t)\right|^{2} \phi(x, t) d x d t \\
& +(1 / 2)\left(\int_{k d}^{k d+d} \int\left|u^{k}(x, t)\right|^{2}\left(D_{t} \phi+\Delta \phi\right)(x, t) d x d t\right) \\
\leqq & -\int_{k d}^{k d+d} \int|D u(x, t)|^{2} \phi(x, t) d x d t \\
& +C\left(2^{6 p}\right)\left(\int_{k d}^{k d+d} \int_{B(a, 2-p)}|u(x, t)|^{2} d x d t\right) .
\end{aligned}
$$

From (4.34), (4.35), Lemma 4.3, the hypothesis of Lemma 4.4, and (4.33) we conclude

$$
\begin{aligned}
& (1 / 2)\left(\int\left|u\left(x, k^{\prime \prime} d+d\right)\right|^{2} \phi\left(x, k^{\prime \prime} d+d\right) d x\right) \\
& -(1 / 2)\left(\int\left|u\left(x, k^{\prime} d\right)\right|^{2} \phi\left(x, k^{\prime} d\right) d x\right)
\end{aligned}
$$




$$
\begin{aligned}
\leqq & C\left(\int_{b-2-2 p}^{b} \int|u(x, t)|^{3}\left(|x-a|+\left(b-t+2^{-2 q}\right)^{1 / 2}\right)^{-5} d x d t\right) \\
& +C d L\left\|H_{\zeta}\right\| 2_{2}^{2}\left(2^{4 q}\right)\left(\int_{b-2^{-2 p}}^{b} \int|D u(x, t)|^{2} d x d t\right) \\
& -\int_{k^{\prime} d}^{k^{\prime \prime} d+d} \int|D u(x, t)|^{2} \phi(x, t) d x d t \\
& +C\left(2^{6 p}\right)\left(\int_{b-2^{-2 p}}^{k^{\prime \prime} d+d} \int_{B\left(a, 2^{-p}\right)}|u(x, t)|^{2} d x d t\right) .
\end{aligned}
$$

Using (4.33), (4.14), Definition 4.1, and the argument that produced (4.35) we obtain

$$
\begin{aligned}
& (1 / 2)\left(\int|u(x, s)|^{2} \phi(x, s) d x\right) \\
& -(1 / 2)\left(\int\left|u\left(x, k^{\prime \prime} d+d\right)\right|^{2} \phi\left(x, k^{\prime \prime} d+d\right) d x\right) \\
= & -\int_{k^{\prime \prime} d+d}^{s} \int|D u(x, t)|^{2} \phi(x, t) d x d t \\
& +(1 / 2)\left(\int_{k^{\prime \prime} d+d}^{s} \int|u(x, t)|^{2}\left(D_{t} \phi+\Delta \phi\right)(x, t) d x d t\right) \\
\leqq & -\int_{k^{\prime \prime} d+d}^{s} \int|D u(x, t)|^{2} \phi(x, t) d x d t \\
& +C\left(2^{6 p}\right)\left(\int_{k^{\prime \prime} d+d}^{s} \int_{B(a, 2-p)}|u(x, t)|^{2} d x d t\right) .
\end{aligned}
$$

From Definition 4.2 and (4.33) we obtain $\beta(t)=0$ if $t \leqq k^{\prime} d$. Hence (4.18) yields

$$
\phi(x, t)=0 \quad \text { if } t \leqq k^{\prime} d .
$$

Combining (4.36), (4.15), (4.37), (4.38), (4.33), and the hypothesis $s \leqq b$ we obtain the conclusion of the lemma.

Lemma 4.5. If $f \in C^{\infty}\left(R^{4}, R^{4}\right), a \in R^{4}$, and $0<r<\infty$ then

$$
\begin{aligned}
& \int_{B(a, r)}|f|^{3} \\
\leqq & C r^{-2}\left(\int_{B(a, 2 r)}|f|^{2}\right)^{3 / 2}+C\left(\int_{B(a, 2 r)}|D f|^{2}\right)\left(\int_{B(a, 2 r)}|f|^{2}\right)^{1 / 2} .
\end{aligned}
$$

Proof. Let $\psi: R^{4} \rightarrow[0,1]$ be a $C^{\infty}$ function such that $\psi(x)=1$ if $x \in B(a, r), \psi(x)=0$ if $x \notin B(a, 2 r)$, and $\|D \psi\|_{\infty} \leqq C r^{-1}$. Applying the argument in Lemma 3.1 to $\psi f$ we find

$$
\begin{aligned}
\int_{B(a, r)}|f|^{3} & \leqq \int|\psi f|^{3} \leqq C\left(\int|D(\psi f)|^{2}\right)\left(\int|\psi f|^{2}\right)^{1 / 2} \\
& \leqq C\left(\|D \psi\|_{\infty}^{2}\left(\int_{B(a, 2 r)}|f|^{2}\right)+\|\psi\|_{\infty}^{2}\left(\int_{B(a, 2 r)}|D f|^{2}\right)\right)\left(\|\psi\|_{\infty}^{2}\left(\int_{B(a, 2 r)}|f|^{2}\right)\right)^{1 / 2} \\
& \leqq C r^{-2}\left(\int_{B(a, 2 r)}|f|^{2}\right)^{3 / 2}+C\left(\int_{B(a, 2 r)}|D f|^{2}\right)\left(\int_{B(a, 2 r)}|f|^{2}\right)^{1 / 2} .
\end{aligned}
$$


Definition 4.6. If $a \in R^{4}, 0<b<\infty, m$ is an integer, and $2^{-2 m} \leqq b$ we set

$$
\begin{aligned}
& A(a, b, m)=2^{m}\left(\int_{b-2^{-2 m}}^{b} \int|u(x, t)|^{3}\left(|x-a|+2^{-m}\right)^{-5} d x d t\right), \\
& B(a, b, m)=2^{6 m}\left(\int_{b-2^{-2 m}}^{b} \int_{B\left(a, 2^{-m}\right)}|u(x, t)|^{3} d x d t\right) .
\end{aligned}
$$

Theorem 4.7. If $a \in R^{4}, 0<b<\infty$, and $p$, $q$ are integers satisfying $p<q, 2^{-2 p} \leqq b$, and $\zeta \leqq 2^{-2 q}$ then

$$
\begin{aligned}
B(a, b, q) \leqq & C(A(a, b, p))+C d^{3 / 2} L^{3}\left\|H_{\zeta}\right\|_{2}^{3}\left(2^{6 q}\right) \\
& +C\left(2^{-p} A(a, b, p)+\sum_{m=p}^{q-1} 2^{-m} B(a, b, m)\right)^{3 / 2}
\end{aligned}
$$

Proof. It is easy to see that Definition 4.6 yields

$$
\begin{aligned}
& \int_{b-2 p}^{b} \int|u(x, t)|^{3}\left(|x-a|+\left(b-t+2^{-2 q}\right)^{1 / 2}\right)^{-5} d x d t \\
\leqq & C\left(2^{-p} A(a, b, p)+\sum_{m=p}^{q-1} 2^{-m} B(a, b, m)\right) .
\end{aligned}
$$

From Hölder's inequality and Definition 4.6 we obtain

$$
\begin{aligned}
& 2^{6 p}\left(\int_{b-2^{-2 p}}^{b} \int_{B(a, 2-p)}|u(x, t)|^{2} d x d t\right) \\
\leqq & 2^{6 p}\left(2^{-6 p} B(a, b, p)\right)^{2 / 3}\left(\int_{b-2^{-2 p}}^{b} \int_{B(a, 2-p)}(1)^{3} d x d t\right)^{1 / 3} \\
\leqq & C(B(a, b, p))^{2 / 3} \leqq C(A(a, b, p))^{2 / 3} .
\end{aligned}
$$

From (4.1), $p<q$, and $\zeta \leqq 2^{-2 q}$ we obtain $d \leqq 2^{-2 p-2}$. Hence $a, b, p, q$ satisfy the properties required in Definition 4.2. Therefore we can define $\phi$ as in Definition 4.2 and use $\zeta \leqq 2^{-2 q}$, Lemma 4.4, (4.39), and (4.40) to conclude

$$
\begin{aligned}
& (1 / 2)\left(\int|u(x, s)|^{2} \phi(x, s) d x\right)+\int_{b-2 p}^{s} \int|D u(x, t)|^{2} \phi(x, t) d x d t \\
\leqq & C\left(2^{-p} A(a, b, p)+\sum_{m=p}^{q-1} 2^{-m} B(a, b, m)\right)+C d L^{2}\left\|H_{\zeta}\right\|_{2}^{2}\left(2^{4 q}\right)+C(A(a, b, p))^{2 / 3}
\end{aligned}
$$

if $b-2^{-2 q} \leqq s \leqq b$. We set

$$
Z=2^{-p} A(a, b, p)+\left(\sum_{m=p}^{q-1} 2^{-m} B(a, b, m)\right)+d L^{2}\left\|H_{\zeta}\right\|_{2}^{2}\left(2^{4 q}\right)+(A(a, b, p))^{2 / 3} .
$$

We consider two cases: $q>p+1$ and $q=p+1$. Assume that $q>p+1$ holds. Definition 4.2 yields

$$
2^{4 q} \leqq C \phi(x, t) \quad \text { if } \quad x \in B\left(a, 2^{-q+1}\right) \quad \text { and } \quad b-2^{-2 q} \leqq t \leqq b .
$$


From (4.41), (4.42), (4.43), and $\phi \geqq 0$ we obtain

$$
\begin{aligned}
& \int_{B\left(a, 2^{-q+1}\right)}|u(x, s)|^{2} d x \leqq C\left(2^{-4 q}\right) Z \quad \text { if } \quad b-2^{-2 q} \leqq s \leqq b, \\
& \int_{b-2^{-2 q}}^{b} \int_{B\left(a, 2^{-q+1}\right)}|D u(x, t)|^{2} d x d t \leqq C\left(2^{-4 q}\right) Z .
\end{aligned}
$$

Since $u$ is $C^{\infty}$ except at the points $(x, k d)$ for $k=0,1,2, \ldots$, Lemma 4.5 yields

$$
\begin{aligned}
& \int_{B\left(a, 2^{-q}\right)}|u(x, s)|^{3} d x \\
\leqq & C\left(2^{2 q}\right)\left(\int_{B\left(a, 2^{-q+1}\right)}|u(x, s)|^{2} d x\right)^{3 / 2} \\
& +C\left(\int_{B\left(a, 2^{-q+1)}\right.}|D u(x, s)|^{2} d x\right)\left(\int_{B\left(a, 2^{-q+1}\right)}|u(x, s)|^{2} d x\right)^{1 / 2}
\end{aligned}
$$

for almost every $s \in\left(b-2^{-2 q}, b\right)$. Now Definition 4.6, (4.44), (4.45), and (4.46) yield

$$
2^{-6 q} B(a, b, q) \leqq C\left(2^{-6 q}\right) Z^{3 / 2} .
$$

From (4.42) we obtain

$$
\begin{aligned}
Z^{3 / 2} \leqq & C\left(2^{-p} A(a, b, p)+\sum_{m=p}^{q-1} 2^{-m} B(a, b, m)\right)^{3 / 2}+C d^{3 / 2} L^{3}\left\|_{\zeta}\right\|_{2}\left(2^{6 q}\right) \\
& +C(A(a, b, p)) .
\end{aligned}
$$

Then (4.47) and (4.48) imply the conclusion of Theorem 4.7 in the case $q>p+1$. Now we assume $q=p+1$. We have

$$
B(a, b, q)=B(a, b, p+1) \leqq C(B(a, b, p)) \leqq C(A(a, b, p)) .
$$

The proof of Theorem 4.7 is complete.

Theorem 4.8. Let $\phi \in C_{0}^{\infty}\left(R^{4} \times R, R^{4}\right)$ such that $\operatorname{div}(\phi)=0$. Let $N$ be a positive real number such that $|D \phi(x, t)-D \phi(x, s)| \leqq N|t-s|$ for all $x \in R^{4}$ and $s, t \in R$. Let $T$ be a positive real number such that $\phi(x, t)=0$ whenever $x \in R^{4}$ and $t \geqq T$. Then

$$
\begin{aligned}
& \quad \mid \int v_{i}(x) \phi_{i}(x, 0) d x+\int_{0}^{\infty} \int u_{i}(x, t)\left(D_{t} \phi_{i}+\Delta \phi_{i}\right)(x, t) d x d t \\
& \quad+\int_{0}^{\infty} \int u_{j}(x, t) u_{i}(x, t) D_{j} \phi_{i}(x, t) d x d t \mid \\
& \leqq C(T+d) L N d+C(T+d)(d+\zeta)^{1 / 2}\|D \phi\|_{\infty}+C(d+\zeta)^{1 / 2} L^{2}\|D \phi\|_{\infty} .
\end{aligned}
$$

Proof. We define $k^{\prime}$ by the properties

$$
k^{\prime} d \leqq T<k^{\prime} d+d, k^{\prime} \text { is an integer. }
$$

Observe that we have $k^{\prime} \geqq 0$. Now let $k \in\left\{0,1,2, \ldots, k^{\prime}\right\}$. We use Definition 4.1 , the semigroup property $H_{a} * H_{b}=H_{a+b}$ (see $[8$, Corollary 1.28, p. 16]) and (4.14) to 
write the following whenever $k d<t<k d+d$ :

$$
\begin{aligned}
w_{i}^{k}(x) & =u_{i}^{k}(x, k d+d)=\left(v_{i}^{k-1} * H_{d}\right)(x) \\
& =\left(\left(v_{i}^{k-1} *(H[t-k d])\right) *(H[k d+d-t])\right)(x) \\
& =\left(u_{i}^{k} *(H[k d+d-t])\right)(x, t)=\left(u_{i} *(H[k d+d-t])\right)(x, t) .
\end{aligned}
$$

From (4.50) and $H_{a} * H_{b}=H_{a+b}$ we conclude

$$
\begin{aligned}
\left(w_{j}^{k} * H_{\zeta}\right)(x) & =\left(\left(u_{j} *(H[k d+d-t])\right) * H_{\zeta}\right)(x, t) \\
& =\left(u_{j} *(H[k d+d-t+\zeta])\right)(x, t)
\end{aligned}
$$

if $k d<t<k d+d$. We set

$$
\begin{aligned}
& H_{t}^{\prime}=H[k d+d-t+\zeta] \quad \text { if } \quad k d<t<k d+d, \\
& H_{t}^{\prime \prime}=H[k d+d-t] \quad \text { if } \quad k d<t<k d+d .
\end{aligned}
$$

Using (4.50), (4.51), (4.52), and (4.53) we obtain

$$
\begin{aligned}
& \int\left(w_{j}^{k} * H_{\zeta}\right)(x) w_{i}^{k}(x) D_{j} \phi_{i}(x, k d+d) d x \\
= & \int\left(u_{j} * H_{t}^{\prime}\right)(x, t)\left(u_{i} * H_{t}^{\prime \prime}\right)(x, t) D_{j} \phi_{i}(x, k d+d) d x
\end{aligned}
$$

if $k d<t<k d+d$. Averaging (4.54) over $t$ we obtain

$$
\begin{aligned}
& \int\left(w_{j}^{k} * H_{\zeta}\right)(x) w_{i}^{k}(x) D_{j} \phi_{i}(x, k d+d) d x \\
= & d^{-1}\left(\int_{k d}^{k d+d} \int\left(u_{j} * H_{t}^{\prime}\right)(x, t)\left(u_{i} * H_{t}^{\prime \prime}\right)(x, t) D_{j} \phi_{i}(x, k d+d) d x d t\right) .
\end{aligned}
$$

From Definition 4.1, (4.10), (3.5), the assumption $\operatorname{div}(\phi)=0$, Definition 3.3, integration by parts, and (4.9) we obtain

$$
\begin{aligned}
& \int u_{i}^{k+1}(x, k d+d) \phi_{i}(x, k d+d) d x \\
= & \int v_{i}^{k}(x) \phi_{i}(x, k d+d) d x \\
= & \int w_{i}^{k}(x) \phi_{i}(x, k d+d) d x-d\left(\int g_{i}^{k}(x) \phi_{i}(x, k d+d) d x\right) \\
= & \int u_{i}^{k}(x, k d+d) \phi_{i}(x, k d+d) d x-d\left(\int f_{i}^{k}(x) \phi_{i}(x, k d+d) d x\right) \\
= & \int u_{i}^{k}(x, k d+d) \phi_{i}(x, k d+d) d x \\
& -d\left(\int\left(w_{j}^{k} * H_{\zeta}\right)(x) D_{j} w_{i}^{k}(x) \phi_{i}(x, k d+d) d x\right) \\
= & \int u_{i}^{k}(x, k d+d) \phi_{i}(x, k d+d) d x \\
& +d\left(\int\left(w_{j}^{k} * H_{\zeta}\right)(x) w_{i}^{k}(x) D_{j} \phi_{i}(x, k d+d) d x\right) .
\end{aligned}
$$

From (4.55) and (4.56) we obtain

$$
\begin{aligned}
& \int u_{i}^{k+1}(x, k d+d) \phi_{i}(x, k d+d) d x \\
& -\int u_{i}^{k}(x, k d+d) \phi_{i}(x, k d+d) d x \\
= & \int_{k d}^{k d+d} \int\left(u_{j} * H_{t}^{\prime}\right)(x, t)\left(u_{i} * H_{t}^{\prime \prime}\right)(x, t) D_{j} \phi_{i}(x, k d+d) d x d t .
\end{aligned}
$$


Taking the inner product of the relation $D_{t}\left(u^{k}\right)=\Delta\left(u^{k}\right)$ with $\phi$ and using (4.14) we obtain

$$
\begin{aligned}
& \int u_{i}^{k}(x, k d+d) \phi_{i}(x, k d+d) d x-\int u_{i}^{k}(x, k d) \phi_{i}(x, k d) d x \\
= & \int_{k d}^{k d+d} \int u_{i}^{k}(x, t)\left(D_{t} \phi_{i}+\Delta \phi_{i}\right)(x, t) d x d t \\
= & \int_{k d}^{k d+d} \int u_{i}(x, t)\left(D_{t} \phi_{i}+\Delta \phi_{i}\right)(x, t) d x d t .
\end{aligned}
$$

Summing (4.57) and (4.58) over all $k \in\left\{0,1,2, \ldots, k^{\prime}\right\}$ and using $u^{0}(x, 0)=v(x),(4.49)$, and the assumption on $T$ we obtain

$$
\begin{aligned}
& -\int v_{i}(x) \phi_{i}(x, 0) d x \\
= & \left(\sum_{k=0}^{k^{\prime}} \int_{k d}^{k d+d} \int\left(u_{j} * H_{t}^{\prime}\right)(x, t)\left(u_{i} * H_{t}^{\prime \prime}\right)(x, t) D_{j} \phi_{i}(x, k d+d) d x d t\right) \\
& +\int_{0}^{\infty} \int u_{i}(x, t)\left(D_{t} \phi_{i}+\Delta \phi_{i}\right)(x, t) d x d t .
\end{aligned}
$$

The Schwarz inequality, Young's inequality, (4.52), (4.53), and (4.16) yield the following if $k d<t<k d+d$ :

$$
\begin{aligned}
& \int\left|\left(u * H_{t}^{\prime}\right)(x, t)\right|\left|\left(u * H_{t}^{\prime \prime}\right)(x, t)\right| d x \\
\leqq & \left(\int\left|\left(u * H_{t}^{\prime}\right)(x, t)\right|^{2} d x\right)^{1 / 2}\left(\int\left|\left(u * H_{t}^{\prime \prime}\right)(x, t)\right|^{2} d x\right)^{1 / 2} \\
\leqq & \left(\int|u(x, t)|^{2} d x\right)^{1 / 2}\left\|H_{t}^{\prime}\right\|_{1}\left(\int|u(x, t)|^{2} d x\right)^{1 / 2}\left\|H_{t}^{\prime \prime}\right\|_{1} \\
= & \int|u(x, t)|^{2} d x \leqq L .
\end{aligned}
$$

From (4.60) and the assumption on $N$ we obtain

$$
\begin{aligned}
& \mid \int_{k d}^{k d+d} \int\left(u_{j} * H_{t}^{\prime}\right)(x, t)\left(u_{i} * H_{t}^{\prime \prime}\right)(x, t) D_{j} \phi_{i}(x, k d+d) d x d t \\
& \quad-\int_{k d}^{k d+d} \int\left(u_{j} * H_{t}^{\prime}\right)(x, t)\left(u_{i} * H_{t}^{\prime \prime}\right)(x, t) D_{j} \phi_{i}(x, t) d x d t \mid \leqq C L N d^{2} .
\end{aligned}
$$

Suppose $k d<t<k d+d$. The Schwarz inequality, the argument in (4.60), Lemma 3.6, (4.52), (4.53), the estimates $k d+d-t<d, k d+d-t+\zeta<d+\zeta$, and (4.16) yield

$$
\begin{aligned}
& \int\left|\left(u_{j} * H_{t}^{\prime}\right)(x, t)\left(u_{i} * H_{t}^{\prime \prime}\right)(x, t)-u_{j}(x, t) u_{i}(x, t)\right| d x \\
& \leqq \int\left|\left(u_{j} * H_{t}^{\prime}\right)(x, t)\left(\left(u_{i} * H_{t}^{\prime \prime}\right)(x, t)-u_{i}(x, t)\right)\right| d x \\
&+\int\left|\left(\left(u_{j} * H_{t}^{\prime}\right)(x, t)-u_{j}(x, t)\right) u_{i}(x, t)\right| d x \\
& \leqq\left(\int\left|\left(u * H_{t}^{\prime}\right)(x, t)\right|^{2} d x\right)^{1 / 2}\left(\int\left|\left(u * H_{t}^{\prime \prime}\right)(x, t)-u(x, t)\right|^{2} d x\right)^{1 / 2} \\
&+\left(\int\left|\left(u * H_{t}^{\prime}\right)(x, t)-u(x, t)\right|^{2} d x\right)^{1 / 2}\left(\int|u(x, t)|^{2} d x\right)^{1 / 2} \\
& \leqq C L^{1 / 2} d^{1 / 2}\left(\int|D u(x, t)|^{2} d x\right)^{1 / 2}+C(d+\zeta)^{1 / 2}\left(\int|D u(x, t)|^{2} d x\right)^{1 / 2} L^{1 / 2} \\
& \leqq C(d+\zeta)^{1 / 2} L^{1 / 2}\left(\int|D u(x, t)|^{2} d x\right)^{1 / 2} \\
&= C\left((d+\zeta)^{1 / 4}\right)\left((d+\zeta)^{1 / 4} L^{1 / 2}\left(\int|D u(x, t)|^{2} d x\right)^{1 / 2}\right) \\
& \leqq C(d+\zeta)^{1 / 2}+C(d+\zeta)^{1 / 2} L\left(\int|D u(x, t)|^{2} d x\right) .
\end{aligned}
$$


From (4.62) we obtain

$$
\begin{aligned}
& \mid \int_{k d}^{k d+d} \int\left(u_{j} * H_{t}^{\prime}\right)(x, t)\left(u_{i} * H_{t}^{\prime \prime}\right)(x, t) D_{j} \phi_{i}(x, t) d x d t \\
& \quad-\int_{k d}^{k d+d} \int u_{j}(x, t) u_{i}(x, t) D_{j} \phi_{i}(x, t) d x d t \mid \\
& \quad \leqq C(d+\zeta)^{1 / 2}\|D \phi\|_{\infty} d+C(d+\zeta)^{1 / 2} L\|D \phi\|_{\infty}\left(\int_{k d}^{k d+d} \int|D u(x, t)|^{2} d x d t\right) .
\end{aligned}
$$

Combining (4.61) and (4.63), summing over $k \in\left\{0,1,2, \ldots, k^{\prime}\right\}$, and using (4.49), the assumption on $T$, and (4.15) we obtain

$$
\begin{aligned}
& \mid\left(\sum_{k=0}^{k^{\prime}} \int_{k d}^{k d+d} \int\left(u_{j} * H_{t}^{\prime}\right)(x, t)\left(u_{i} * H_{t}^{\prime \prime}\right)(x, t) D_{j} \phi_{i}(x, k d+d) d x d t\right) \\
& \quad-\int_{0}^{\infty} \int u_{j}(x, t) u_{i}(x, t) D_{j} \phi_{i}(x, t) d x d t \mid \\
& \leqq C\left(k^{\prime}+1\right) L N d^{2}+C\left(k^{\prime}+1\right)(d+\zeta)^{1 / 2}\|D \phi\|_{\infty} d \\
& \quad+C(d+\zeta)^{1 / 2} L\|D \phi\|_{\infty}\left(\int_{0}^{\infty} \int|D u(x, t)|^{2} d x d t\right) \\
& \leqq C\left(k^{\prime} d+d\right) L N d+C\left(k^{\prime} d+d\right)(d+\zeta)^{1 / 2}\|D \phi\|_{\infty}+C(d+\zeta)^{1 / 2} L^{2}\|D \phi\|_{\infty} \\
& \leqq C(T+d) L N d+C(T+d)(d+\zeta)^{1 / 2}\|D \phi\|_{\infty}+C(d+\zeta)^{1 / 2} L^{2}\|D \phi\|_{\infty} .
\end{aligned}
$$

Finally, (4.59) and (4.64) yield the conclusion of Theorem 4.8.

Lemma 4.9. If $\phi \in C_{0}^{\infty}\left(R^{4} \times R, R\right)$ then $\int_{0}^{\infty} \int u_{i}(x, t) D_{i} \phi(x, t) d x d t=0$.

Proof. This follows from (4.2), the definition of $u^{k}$, and (4.14).

Lemma 4.10. If $0<\eta<\infty, a \in R^{4}$, and $0 \leqq t^{\prime} \leqq t^{\prime \prime}<\infty$ then

$$
\left|\left(u * H_{\eta}\right)\left(a, t^{\prime \prime}\right)-\left(u * H_{\eta}\right)\left(a, t^{\prime}\right)\right| \leqq C\left(d+\left(t^{\prime \prime}-t^{\prime}\right)\right)\left(L^{1 / 2} \eta^{-2}+L \eta^{-5 / 2}\right) .
$$

Proof. Define $k^{\prime}$ and $k^{\prime \prime}$ by

$$
k^{\prime} d \leqq t^{\prime}<k^{\prime} d+d, k^{\prime \prime} d \leqq t^{\prime \prime}<k^{\prime \prime} d+d, k^{\prime} \text { and } \quad k^{\prime \prime} \quad \text { are integers } .
$$

We clearly have $0 \leqq k^{\prime} \leqq k^{\prime \prime}$. From (4.14) and Definition 4.1 we obtain

$$
\left(u * H_{\eta}\right)\left(a, k^{\prime} d\right)=\left(u^{k^{\prime}} * H_{\eta}\right)\left(a, k^{\prime} d\right)=\left(v^{k^{\prime}-1} * H_{\eta}\right)(a) .
$$

If $k^{\prime} d<t^{\prime}$ then (4.65), (4.14), Definition 4.1, and the semigroup property $H_{a+b}$ $=H_{a} * H_{b}$ yield

$$
\begin{aligned}
& \left(u * H_{\eta}\right)\left(a, t^{\prime}\right)=\left(u^{k^{\prime}} * H_{\eta}\right)\left(a, t^{\prime}\right)=\left(\left(v^{k^{\prime}-1} * H\left[t^{\prime}-k^{\prime} d\right]\right) * H_{\eta}\right)(a) \\
& =\left(v^{k^{\prime}-1} * H\left[t^{\prime}-k^{\prime} d+\eta\right]\right)(a) \text { if } k^{\prime} d<t^{\prime} .
\end{aligned}
$$


Now (4.66), (4.67), the Schwarz inequality, (4.2), and (4.65) yield

$$
\begin{aligned}
\mid(u & \left.* H_{\eta}\right)\left(a, t^{\prime}\right)-\left(u * H_{\eta}\right)\left(a, k^{\prime} d\right) \mid \\
& =\left|\left(v^{k^{\prime}-1} *\left(H\left[t^{\prime}-k^{\prime} d+\eta\right]-H[\eta]\right)\right)(a)\right| \\
& \leqq\left\|v^{k^{\prime}-1}\right\|_{2}\left\|H\left[t^{\prime}-k^{\prime} d+\eta\right]-H[\eta]\right\|_{2} \\
& \leqq C L^{1 / 2}\left(t^{\prime}-k^{\prime} d\right) \eta^{-2} \leqq C L^{1 / 2} d \eta^{-2}
\end{aligned}
$$

A similar computation yields

$$
\left|\left(u * H_{\eta}\right)\left(a, t^{\prime \prime}\right)-\left(u * H_{\eta}\right)\left(a, k^{\prime \prime} d\right)\right| \leqq C L^{1 / 2} d \eta^{-2} .
$$

Now we fix an integer $k$ satisfying $k \geqq 0$. The arguments that yielded (4.66), (4.67), and (4.68) also yield

$$
\begin{aligned}
& \left|\left(u^{k} * H_{\eta}\right)(a, k d)-\left(u^{k} * H_{\eta}\right)(a, k d+d)\right| \\
& \quad=\left|\left(v^{k-1} * H_{\eta}\right)(a)-\left(v^{k-1} * H[d+\eta]\right)(a)\right| \leqq C L^{1 / 2} d \eta^{-2} .
\end{aligned}
$$

From Definition 4.1 [in particular (4.10)] we obtain

$$
\begin{aligned}
& \left(u^{k+1} * H_{\eta}\right)(a, k d+d)-\left(u^{k} * H_{\eta}\right)(a, k d+d) \\
& \quad=\left(v^{k} * H_{\eta}\right)(a)-\left(w^{k} * H_{\eta}\right)(a)=-d\left(g^{k} * H_{\eta}\right)(a) .
\end{aligned}
$$

We fix $n \in\{1,2,3,4\}$ and define $\phi: R^{4} \rightarrow R^{4}$ by $\phi_{n}(x)=H_{\eta}(a-x), \phi_{i}(x)=0$ if $i \neq n$. Then approximation of $\phi$ by functions with compact support, (3.5), Definition 3.3, (4.7), (4.8), integration by parts, (4.9), (1.5), Young's inequality, (1.3), and (4.7) yield

$$
\begin{aligned}
& \left|\left(g_{n}^{k} * H_{\eta}\right)(a)\right|=\left|\int g_{n}^{k}(x) H_{\eta}(a-x) d x\right| \\
& \quad=\left|\int g_{i}^{k}(x) \phi_{i}(x) d x\right|=\left|\int f_{i}^{k}(x)\left(\phi_{i}-\left(\left(D_{i} \operatorname{div}(\phi)\right) * K\right)\right)(x) d x\right| \\
& \quad=\left|\int\left(w_{j}^{k} * H_{\zeta}\right)(x) D_{j} w_{i}^{k}(x)\left(\phi_{i}-\left(\left(D_{i} \operatorname{div}(\phi)\right) * K\right)\right)(x) d x\right| \\
& \quad=\left|\int\left(w_{j}^{k} * H_{\zeta}\right)(x) w_{i}^{k}(x)\left(D_{j} \phi_{i}-\left(\left(D_{i j} \operatorname{div}(\phi)\right) * K\right)\right)(x) d x\right| \\
& \quad \leqq C\left\|_{w^{k}} * H_{\zeta}\right\|_{2}\left\|w^{k}\right\|_{2} \eta^{-5 / 2} \leqq C\left\|_{w^{k}}\right\|_{2}\left\|H_{\zeta}\right\|_{1}\left\|w^{k}\right\|_{2} \eta^{-5 / 2} \leqq C L \eta^{-5 / 2} .
\end{aligned}
$$

Now (4.71) and (4.72) yield

$$
\left|\left(u^{k+1} * H_{\eta}\right)(a, k d+d)-\left(u^{k} * H_{\eta}\right)(a, k d+d)\right| \leqq C L d \eta^{-5 / 2} .
$$

From (4.70), (4.73), and (4.14) we obtain

$$
\left|\left(u * H_{\eta}\right)(a, k d)-\left(u * H_{\eta}\right)(a,(k+1) d)\right| \leqq C L^{1 / 2} d \eta^{-2}+C L d \eta^{-5 / 2} .
$$

From (4.68), (4.69), and (4.74) we obtain

$$
\begin{aligned}
& \left|\left(u * H_{\eta}\right)\left(a, t^{\prime \prime}\right)-\left(u * H_{\eta}\right)\left(a, t^{\prime}\right)\right| \\
& \quad \leqq C L^{1 / 2} d \eta^{-2}+\left(k^{\prime \prime}-k^{\prime}\right)\left(C L^{1 / 2} d \eta^{-2}+C L d \eta^{-5 / 2}\right) .
\end{aligned}
$$

The conclusion of Lemma 4.10 follows from (4.75) and the inequality $\left(k^{\prime \prime}-k^{\prime}\right) d$ $\leqq\left(t^{\prime \prime}-t^{\prime}\right)+d$. 
Lemma 4.11. If $0<\eta<\infty, a \in R^{4}, 0 \leqq t<\infty$, and $\delta>0$ then

$$
\begin{aligned}
& \left|\left(u * H_{\eta}\right)(a, t)-(1 / \delta)\left(\int_{t}^{t+\delta}\left(u * H_{\eta}\right)(a, s) d s\right)\right| \\
& \leqq C(d+\delta)\left(L^{1 / 2} \eta^{-2}+L \eta^{-5 / 2}\right) .
\end{aligned}
$$

Proof. This follows immediately from Lemma 4.10.

\section{Section 5. Passage to the Limit}

Definition 5.1. We choose infinite sequences $\zeta_{1}, \zeta_{2}, \zeta_{3}, \ldots$ and $d_{1}, d_{2}, d_{3}, \ldots$ of positive real numbers satisfying $d_{n} \leqq \zeta_{n}, \quad d_{n} L\left\|H_{\zeta_{n}}\right\|_{2}^{2} \leqq 1, \quad \lim _{n \rightarrow \infty} \zeta_{n}=0$, and $\lim _{n \rightarrow \infty} d_{n}\left\|H_{\zeta_{n}}\right\|_{2}^{2}=0$. For each $n$ we define the function $(u, n): R^{4} \times[0, \infty) \rightarrow R^{4}$ by $(u, n)$ $=u$ where $u$ is the function obtained as in Section 4 using $\zeta=\zeta_{n}$ and $d=d_{n}$ [Definition 4.1 and (4.14)]. From (4.17) we obtain

$$
\int_{0}^{\infty} \int|(u, n)(x, t)|^{3} d x d t \leqq C L^{3 / 2}
$$

Hence, by passing to a subsequence, we may assume that there is a function $u \in L^{3}\left(R^{4} \times[0, \infty), R^{4}\right)$ such that

$u$ is the weak limit of $(u, n)$ in $L^{3}$.

Lemma 5.2. Let $0<T<\infty$. Then

$$
\lim _{n \rightarrow \infty} \int_{0}^{T} \int_{B(0, T)}|(u, n)(x, t)-u(x, t)|^{2} d x d t=0
$$

Proof. Suppose $\varepsilon>0$ is given. Let $\eta$ be a positive real number such that $\eta^{1 / 2} L^{1 / 2} \leqq \varepsilon$. Let $\delta$ be a positive real number such that $\delta\left(L^{1 / 2} \eta^{-2}+L \eta^{-5 / 2}\right) T^{5 / 2} \leqq \varepsilon$. Define $f_{n}: R^{4} \times[0, \infty) \rightarrow R^{4}$ and $f: R^{4} \times[0, \infty) \rightarrow R^{4}$ by

$$
\begin{aligned}
& f_{n}(a, t)=(1 / \delta)\left(\int_{t}^{t+\delta}\left((u, n) * H_{\eta}\right)(a, s) d s\right), \\
& f(a, t)=(1 / \delta)\left(\int_{t}^{t+\delta}\left(u * H_{\eta}\right)(a, s) d s\right) .
\end{aligned}
$$

Then (5.1) implies that the sequence $f_{n}$ is equicontinuous. From (5.2) we conclude $\lim _{n \rightarrow \infty} f_{n}(a, t)=f(a, t)$ for all $(a, t)$. Therefore $f_{n}$ converges to $f$ uniformly on the compact set $B(0, T) \times[0, T]$, and hence

$$
\lim _{n \rightarrow \infty} \int_{0}^{T} \int_{B(0, T)}\left|f_{n}(x, t)-f(x, t)\right|^{2} d x d t=0 .
$$


If $n$ is a positive integer then Minkowski's inequality, Lemmas 3.6, 4.11, (4.15), and the choice of $\eta$ and $\delta$ yield

$$
\begin{aligned}
&\left(\int_{0}^{T} \int_{B(0, T)}\left|(u, n)(x, t)-f_{n}(x, t)\right|^{2} d x d t\right)^{1 / 2} \\
& \leqq\left(\int_{0}^{\infty} \int\left|(u, n)(x, t)-\left((u, n) * H_{\eta}\right)(x, t)\right|^{2} d x d t\right)^{1 / 2} \\
& \quad+\left(\int_{0}^{T} \int_{B(0, T)}\left|\left((u, n) * H_{\eta}\right)(x, t)-f_{n}(x, t)\right|^{2} d x d t\right)^{1 / 2} \\
& \leqq\left(\int_{0}^{\infty} C \eta\left(\int|D(u, n)(x, t)|^{2} d x\right) d t\right)^{1 / 2} \\
&+\left(\int_{0 B(0, T)}^{T}\left(C\left(d_{n}+\delta\right)\left(L^{1 / 2} \eta^{-2}+L \eta^{-5 / 2}\right)\right)^{2} d x d t\right)^{1 / 2} \\
& \leqq C \eta^{1 / 2} L^{1 / 2}+C\left(d_{n}+\delta\right)\left(L^{1 / 2} \eta^{-2}+L \eta^{-5 / 2}\right) T^{5 / 2} \\
& \leqq C \varepsilon+C d_{n}\left(L^{1 / 2} \eta^{-2}+L \eta^{-5 / 2}\right) T^{5 / 2} .
\end{aligned}
$$

Since $\lim d_{n}=0$, we can choose a positive integer $N$ such that $d_{n}\left(L^{1 / 2} \eta^{-2}\right.$ $\left.+L \eta^{-5 / 2}\right) T^{5 / 2} \leqq \varepsilon$ if $n \geqq N$. We may assume [using (5.3)] that

$$
\left(\int_{0}^{T} \int_{B(0, T)}\left|f_{n}(x, t)-f_{N}(x, t)\right|^{2} d x d t\right)^{1 / 2} \leqq \varepsilon \quad \text { if } \quad n \geqq N .
$$

Hence (5.4) implies

$$
\left(\int_{0}^{T} \int_{B(0, T)}|(u, n)(x, t)-(u, N)(x, t)|^{2} d x d t\right)^{1 / 2} \leqq C \varepsilon \quad \text { if } \quad n \geqq N .
$$

From (5.2) we conclude that the restriction of $u$ to $B(0, T) \times(0, T)$ is the weak limit in $L^{2}$ of the restrictions of the $(u, n)$ to $B(0, T) \times(0, T)$. Hence $(5.5)$ and the fact that the unit ball of $L^{2}$ is weakly closed imply

$$
\left(\int_{0}^{T} \int_{B(0, T)}|u(x, t)-(u, N)(x, t)|^{2} d x d t\right)^{1 / 2} \leqq C \varepsilon .
$$

Now (5.5) and (5.6) imply the conclusion of the lemma.

Theorem 5.3. The function $u$ is a weak solution to the Navier-Stokes equations of incompressible fluid flow with initial condition $v$.

Proof. From Definition 5.1 we obtain $u \in L^{3}\left(R^{4} \times[0, \infty), R^{4}\right)$. Let $\phi \in C_{0}^{\infty}\left(R^{4} \times R, R\right)$. Then Lemma 4.9 and (5.2) yield (1.1). Now let $\phi \in C_{0}^{\infty}\left(R^{4} \times R, R^{4}\right)$ with $\operatorname{div}(\phi)=0$. Let $N$ be as in Theorem 4.8 and let $T$ be a positive real number such that $\operatorname{spt}(\phi) \cap\left(R^{4} \times[0, \infty)\right) \subset B(0, T) \times[0, T]$. Then Lemma 5.2, Theorem 4.8, $\lim _{n \rightarrow \infty} d_{n}=0$, and $\lim _{n \rightarrow \infty} \zeta_{n}=0$ imply (1.2).

We can now finish the proof of Theorem 1.1. We set $f_{n}=(u, n)$ and $f=u$ (Definition 5.1). Then (5.1) and (5.2) imply that there exists a positive real number 
$D$ such that the conditions in the first paragraph of Section 2 are satisfied. From Definitions 5.1, 4.6, and Theorem 4.7 we conclude that there exists $0<M<\infty$ such that property $P(M)$ holds (see Definitions 2.2 and 2.1). Let $\varepsilon>0$ correspond to $M$ as in Lemma 2.3. Then Lemma 2.9 implies that there exist sets $A_{p}$ satisfying 1), 2), and 3) of Lemma 2.9. From parts 1), 3) of that lemma, the fact $f=u$, Theorem 5.3, and the argument at the end of Section 2 in [5], we conclude that the restriction of $u$ to $\left(R^{4} \times\left(3\left(2^{-2 p}\right), \infty\right)\right)-A_{p}$ is equal almost everywhere to a continuous function. Hence, by modifying $u$ on a set of Lebesgue measure zero and setting

$$
A=\bigcap_{p=0}^{\infty}\left(A_{p} \cup\left(R^{4} \times\left(0,3\left(2^{-2 p}\right)\right]\right)\right)
$$

we can conclude that the restriction of $u$ to $\left(R^{4} \times(0, \infty)\right)-A$ is continuous. This proves part c) of Theorem 1.1. From part 1) of Lemma 2.9 we conclude that part a) of Theorem 1.1 holds. Let $p \geqq 0$ be an integer and let $\delta>0$ be given. There exists an integer $q$ such that $p \leqq q$ and $5^{1 / 2} 2^{-q} \leqq \delta$. From

$$
\begin{aligned}
& A \cap\left(R^{4} \times\left(3\left(2^{-2 p}\right), \infty\right)\right) \\
& \quad C\left(A_{q} \cup\left(R^{4} \times\left(0,3\left(2^{-2 q}\right)\right]\right)\right) \cap\left(R^{4} \times\left(3\left(2^{-2 p}\right), \infty\right)\right) \subset A_{q},
\end{aligned}
$$

Definition 2.8, and part 2) of Lemma 2.9 we conclude

$$
\phi_{\delta}\left(A \cap\left(R^{4} \times\left(3\left(2^{-2 p}\right), \infty\right)\right)\right) \leqq \phi_{\delta}\left(A_{q}\right) \leqq C \varepsilon^{-1} D .
$$

Hence Definition 2.8 yields $H^{3}\left(A \cap\left(R^{4} \times\left(3\left(2^{-2 p}\right), \infty\right)\right)\right) \leqq C \varepsilon^{-1} D$. Since $H^{3}$ is a Borel measure, we can use $A \subset R^{4} \times(0, \infty)$ to conclude $H^{3}(A) \leqq C \varepsilon^{-1} D$. This proves part b) of Theorem 1.1. From Theorem 5.3 and the fact $u \in L^{3}\left(R^{4} \times[0, \infty), R^{4}\right)$ (see Definition 5.1) we obtain the remaining conclusions of Theorem 1.1.

\section{References}

1. Almgren,F.J., Jr.: Existence and regularity almost everywhere of solutions to elliptic variational problems with constraints. Memoirs of the American Mathematical Society 165. Providence, R.I.: American Mathematical Society 1976

2. Federer,H.: Geometric measure theory. Berlin-Heidelberg-New York: Springer 1969

3. Mandelbrot, B. : Intermittent turbulence and fractal dimension kurtosis and the spectral exponent $5 / 3+B$. In: Turbulence and Navier-Stokes equation. Lecture notes in mathematics. Vol. 565. BerlinHeidelberg-New York: Springer 1976

4. Scheffer, V.: Hausdorff measure and the Navier-Stokes equations. Commun. math. Phys. 55, 97$112(1977)$

5. Scheffer, V.: Partial regularity of solutions to the Navier-Stokes equations. Pacific J. Math. 66. No. 2, 535-552 (1976)

6. Scheffer,V.: Turbulence and Hausdorff dimension. In: Turbulence and Navier-Stokes equation. Lecture notes in mathematics. Vol. 565. Berlin-Heidelberg-New York: Springer 1976

7. Stein,E.M.: Singular integrals and differentiability properties of functions. Princeton: Princeton University Press 1970

8. Stein, E.M., Weiss, G.L. : Introduction to fourier analysis on euclidean spaces. Princeton : Princeton University Press 1971

Communicated by J. Glimm

Received January 17, 1978 